Revue des patrimoines

29 | 2016

Ensembles mobiliers, industriels, techniques.

Connaissance, protection, conservation, présentation au public

\title{
Fontainebleau, la restauration des tentures brodées du Salon jaune de Joséphine ou le parti- pris de l'authenticité
}

\section{Vincent Cochet et Agathe Strouk}

\section{(Q) OpenEdition \\ Journals}

Édition électronique

URL : http://journals.openedition.org/insitu/13060

DOI : 10.4000/insitu.13060

ISSN : 1630-7305

Éditeur

Ministère de la culture

Référence électronique

Vincent Cochet et Agathe Strouk, « Fontainebleau, la restauration des tentures brodées du Salon jaune de Joséphine ou le parti-pris de l'authenticité », In Situ [En ligne], 29 | 2016, mis en ligne le 07

septembre 2016, consulté le 19 avril 2019. URL : http://journals.openedition.org/insitu/13060 ; DOI :

10.4000/insitu. 13060

Ce document a été généré automatiquement le 19 avril 2019

In Situ Revues des patrimoines est mis à disposition selon les termes de la licence Creative Commons Attribution - Pas d'Utilisation Commerciale - Pas de Modification 4.0 International. 


\title{
Fontainebleau, la restauration des tentures brodées du Salon jaune de Joséphine ou le parti-pris de l'authenticité
}

\author{
Vincent Cochet et Agathe Strouk
}

1 Occupé sans discontinuité depuis François I ${ }^{\text {er }}$, le château de Fontainebleau fut l'une des résidences les plus prisées des rois de France et des empereurs. Il constitue un véritable foyer artistique à la Renaissance et l'écrin de somptueux aménagements sous les Bourbons, jusqu'à la veille de la Révolution française. Néanmoins, le Premier Empire marque le palais d'une empreinte éclatante. L'ampleur des transformations effectuées à cette époque n'a aucun rapport avec la durée et la fréquence des séjours de Napoléon I ${ }^{\text {er }}$, mais cette revitalisation signe l'avenir du château. En effet, l'antique château connaît une métamorphose dès 1804, lorsque l'Empereur décide d'y accueillir le pape Pie VII, venu de Rome pour la cérémonie du sacre à Notre-Dame de Paris. Ce séjour de quelques jours est déterminant pour Fontainebleau, qui retrouve son lustre d'antan. En l'espace d'un mois, les appartements sont remeublés à la hâte et préparés pour recevoir le couple impérial, les princes, les grands dignitaires et le souverain pontife. Napoléon s'installe dans les appartements des rois de France et l'impératrice Joséphine prend possession de ceux de Marie-Antoinette.

\section{Joséphine en ses Petits Appartements}

2 La vie de cour instaurée par la création de l'Empire fait de Fontainebleau l'une des résidences des souverains. Élaborée en 1805, l'étiquette des palais impériaux prévoit une division très nette entre les appartements de représentation et les appartements ordinaires des souverains ${ }^{1}$. Les premiers sont situés au premier étage du palais, dans les anciens appartements des rois de France. Les seconds, envisagés dès 1805, sont progressivement aménagés au cours du régime. Pour l'impératrice, ils sont censés 
comporter un appartement d'honneur et un appartement intérieur. Une salle des gardes, un premier salon, un second salon - le salon de l'Impératrice - une salle à manger et une salle de concert composent l'appartement d'honneur. Les pièces intérieures sont un salon, la chambre à coucher, le cabinet de toilette et un boudoir. Tributaire des lieux, cette partition ne peut être rigoureusement appliquée à Fontainebleau, résidence secondaire qui ne peut rivaliser avec les Tuileries, d'autant que l'Empereur se réserve plusieurs pièces pour les bureaux de ses secrétaires au détriment des espaces de l'Impératrice. Hors les services et les atours, ces nouveaux appartements comportent neuf pièces (fig. 1).

Figure 1

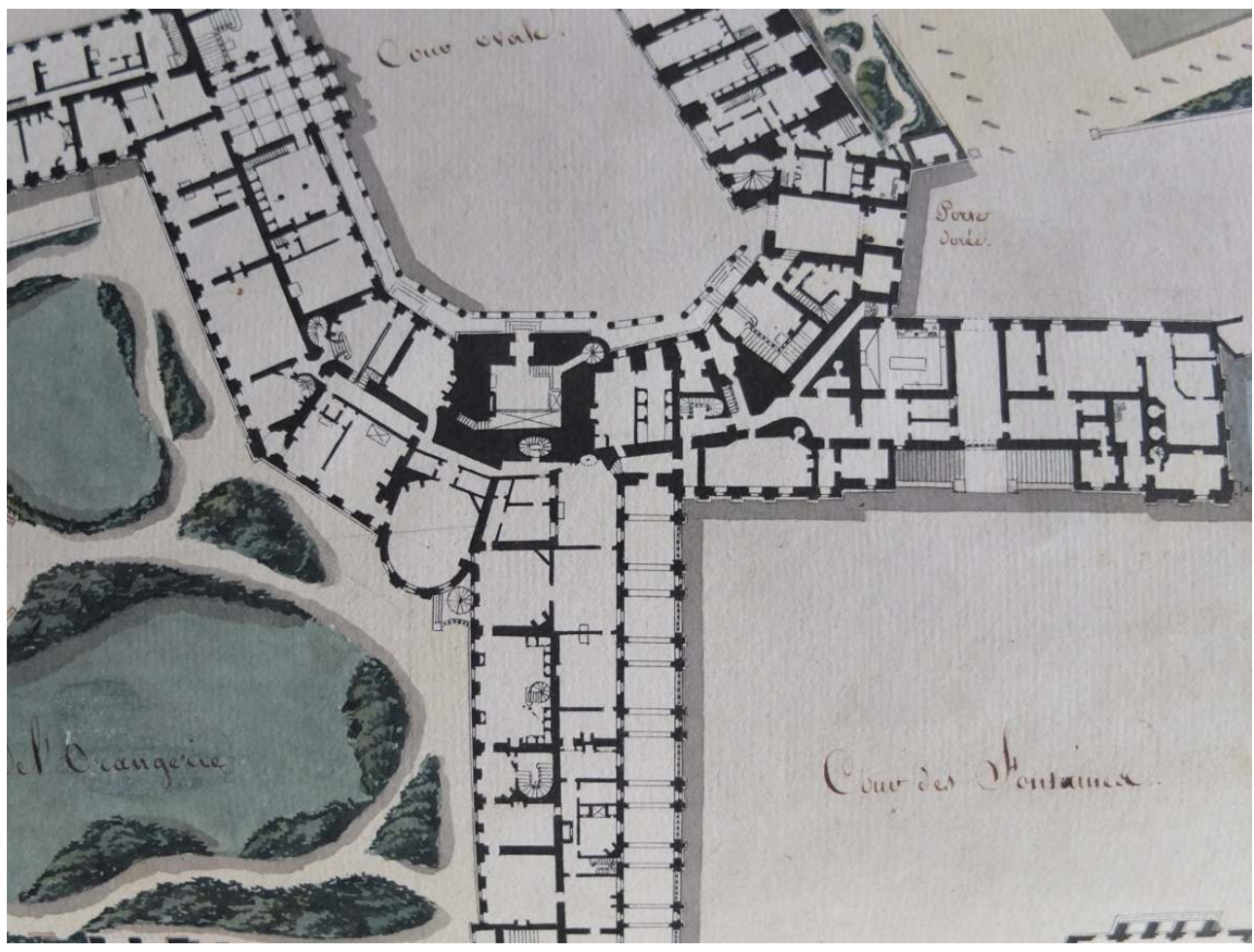

Atlas de 1811, plan du rez-de-chaussée (détail), avec les Petits Appartements de l'Impératrice. Depuis la Cour ovale (en haut à gauche), un vestibule permet d'accéder à l'antichambre des Petits Appartements de l'Impératrice, éclairée sur le jardin de l'Orangerie. Celle-ci débute l'enfilade de ces appartements qui comprend successivement une pièce intermédiaire, le salon de billard, le deuxième ou Grand Salon (Salon jaune), le salon d'attente des dames d'honneur, un petit passage ménagé dans l'angle que forme la façade sur le jardin, la salle de bains, avec sa garde-robe située à l'arrière, la chambre, un petit boudoir et enfin, dans la pièce en rotonde, la salle à manger dite salon d'étude. Fontainebleau, château (archives).

Phot. Cochet, Vincent, 2014. (c) Vincent Cochet.

\section{Les broderies du Grand Salon}

Le deuxième et Grand Salon des Petits Appartements de l'Impératrice se situe à l'emplacement du grand cabinet du Dauphin et du duc de Normandie, fils de Louis XVI. Son volume résulte de la réunion en 1786 de deux pièces contiguës des appartements de Mesdames, filles de Louis XV, puis de Madame Royale. En 1808, ce vaste salon, éclairé par quatre grandes fenêtres ouvrant au rez-de-chaussée sur le jardin de l'Orangerie, actuel jardin de Diane, est la pièce d'assemblée, où l'Impératrice reçoit les princes et princesses 
de la famille impériale, les grands officiers de l'Empire et leurs épouses, les dames d'honneur, les chambellans, l'écuyer de service ou encore la dame d'atours, les officiers et aides de camp de l'empereur.

Le salon fait l'objet d'importants travaux sous la conduite de l'architecte Antoine Leroy (dates inconnues) en $1808^{2}$. Les parquets, les lambris, les plâtres et la cheminée sont repris. Trois miroirs sont insérés dans les entre-fenêtres et une glace marque le centre de chaque paroi. La relative simplicité de ces aménagements en serait atténuée "si le meuble en étoffe qui pourra être employé et choisi favorisait la richesse de l'ameublement du salon, en même temps qu'il pourrait présenter le moyen de faire 6 pièces de tenture régulières et parallèles ", selon le vœu de Napoléon ${ }^{3}$. Le menuisier Leclerc (dates inconnues) crée et installe les bordures des glaces et des tapisseries qui sont immédiatement déposées pour les faire dorer à Paris, tandis que les châssis de tenture sont confectionnés en chêne des Vosges ${ }^{4}$. Mais, confronté au mauvais état de la menuiserie ancienne, l'architecte entreprend des travaux dont le coût excède le budget alloué. D'où une sévère remontrance de l'intendant général de la Maison de l'empereur ${ }^{5}$. Pressé par l'annonce d'un voyage à Fontainebleau en octobre 1808, Leroy ne sut endiguer les débordements. Le 26 juillet, Duroc, grand maréchal du palais, rappelle cependant que «l'on pouvait, ainsi que cela avait été proposé, laisser en suspend l'ameublement des Petits Appartements de S.M. l'Impératrice, d'autant plus que l'on n'a pas ses instructions et qu'ainsi on risque de faire des choses qui ne lui conviendraient pas $^{6}{ }^{6}$.

En effet, il s'agit de répondre aux désirs de Joséphine qui impose Michel-Jacques Boulard (1761-1825), tapissier qui participa à la décoration de son hôtel parisien sous le Consulat et fut de nouveau appelé en 1806 et en 1808 pour réaliser les tentures en velours de soie brodé d'or de la grande chambre d'honneur de l'Impératrice aux Tuileries et de sa chambre à Saint-Cloud.

Le 4 décembre 1808, Boulard soumet son devis pour le meuble d'étoffe du Grand Salon ${ }^{7}$. Le compartimentage établi par Leroy régit la disposition du décor textile et du mobilier, dont l'emplacement répond aussi à une bouche de chaleur. En effet, un poêle, placé à l'arrière du mur faisant face aux fenêtres, commande l'installation d'une console « composée en métal ou garnie de manière à être incombustible. Ce meuble sera de la largeur de six pieds au moins, mesure qui doit être d'accord avec celle de la glace et parquets observant les mêmes chants que les autres glaces pour le placement de trois paires de bras en bronze doré, objet qui détermine le placement des deux canapés ${ }^{8}$ ». Les deux «canapés en divans » de 3,20 mètres de large prennent place le long des murs, couverts de huit pièces de tenture en $15 / 16$ jaune «brodées au passé. Façon de 8 pièces de tenture faite selon les plans ainsi que pour la disposition des broderies ${ }^{9}$. Celles-ci sont posées en 1809 (fig. 2). Leur répartition reste hypothétique car les soumissions et mémoires du tapissier n'indiquent qu'une quantité de 15/16 jaune $(41,60 \mathrm{~m})$ ou la longueur totale finie pour les huit pièces, sans donner la largeur de chaque panneau. 


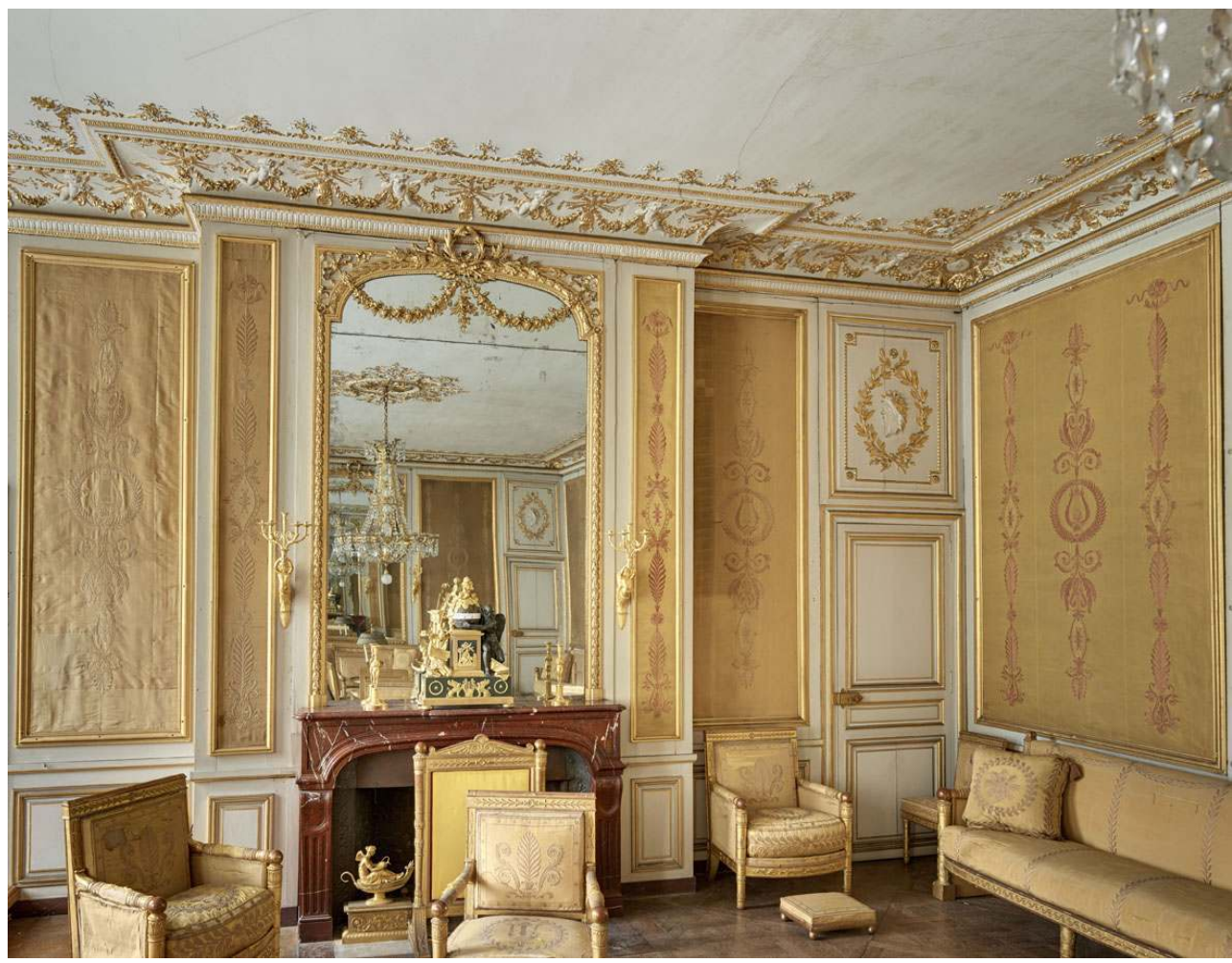

Vue d'ensemble du Salon jaune (état actuel). 14-542136, Fontainebleau, château.

Phot. Didierjean, Adrien, 2014. (c) RMN-Grand Palais (Château de Fontainebleau).

Il est vraisemblable que les huit pièces de tenture se répartissaient de la manière suivante : une de chaque côté des miroirs de la cheminée et du mur en vis-à-vis, et quatre panneaux sur le grand mur face aux fenêtres. Inventoriée en 1810, la tenture est dite à «broderie amaranthe, dessin véla, palmes, lyres et abeilles, doublées en toile Laval blanche. $15 \mathrm{~m} 08$ de cours sur $2 \mathrm{~m} 80$ de haut ».

Pour chacun des panneaux, les ornements se répétaient selon des séquences particulières; la disposition dans la pièce faisait certainement alterner les différents motifs. Ce sont tout d'abord des pilastres composés d'un culot à enroulements d'où s'échappent quatre palmes séparées par des rosettes avec, au centre, une mandorle autrefois brodée d'une abeille et, au sommet, une rosette d'où flottent des rubans. Simples ou doublés, ces pilastres encadrent des panneaux de largeur moyenne (environ $90 \mathrm{~cm}$ ), brodés de deux motifs différents. L'un présente une lyre enserrée dans une couronne de laurier d'où se développent, vers le haut et de manière symétrique vers le bas, des volutes donnant naissance à des palmes encadrant un culot, prolongé par une mandorle, puis par des feuilles d'où jaillit une palmette (fig. 3). Sur l'autre, une couronne de laurier dans laquelle deux trompes sont disposées en sautoir surmonte un grand panier de fleurs reposant sur un thyrse fleuronné, sous lequel se déploie en éventail un velum doublé d'une guirlande de palmettes. Au centre de celle-ci, un pendentif à rosette jouxte en partie basse un grand culot à palmes formant enroulements et timbré d'une palmette (fig. 4). 
Figure 3

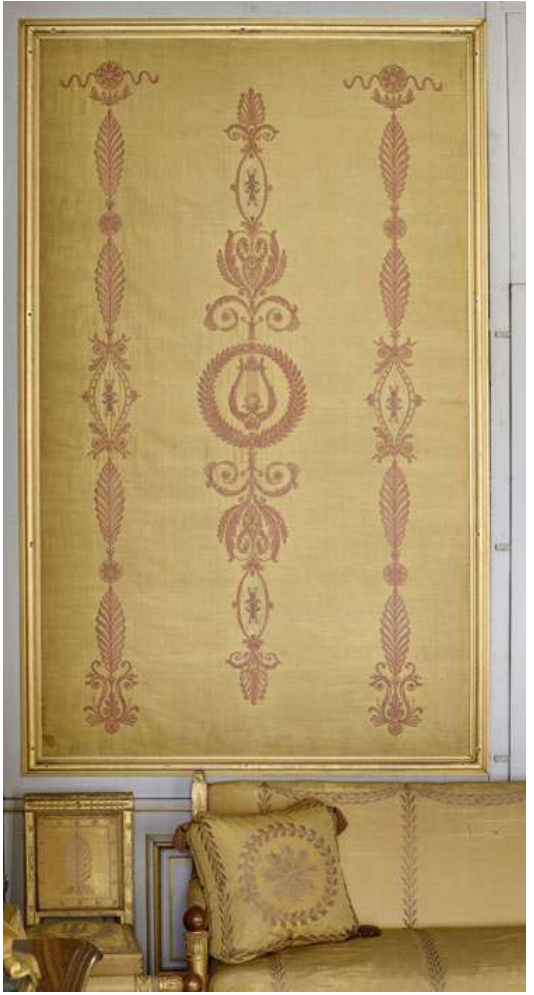

Deuxième panneau traité, vue frontale. 14-542139, Fontainebleau, château.

Phot. Didierjean, Adrien, 2014. (C) RMN-Grand Palais (Château de Fontainebleau). 
Figure 4

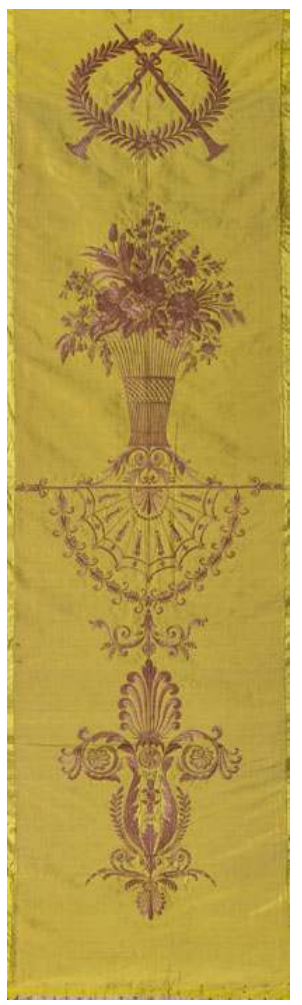

Grand panneau à bouquet et velum (inv. F 2598.2.1). 14-543446, hauteur : 2,8 $\mathrm{m}$ x largeur : 1,4 m, Fontainebleau, château.

Phot. Didierjean, Adrien, 2014. (c) RMN-Grand Palais (Château de Fontainebleau).

Montées sous des moulures dorées par l'entrepreneur Chaise (dates inconnues), ces tentures sont réalisées au point passé, technique de broderie relativement simple, avec un fil de soie retors, suivant un dessin à l'encre sur le taffetas. L'orientation des points permet de créer des effets de dessin et de relief. Les raccords de lés, dissimulés sous les motifs, indiquent que la distribution du dessin est faite en fonction des dimensions de chaque panneau. Le répertoire ornemental combine des motifs employés de manière récurrente dans les arts décoratifs du premier quart du xix $x^{e}$ siècle, en particulier le panier fleuri ou encore la lyre dans une couronne végétale, dont le Recueil de décorations intérieures publié par Percier et Fontaine en 1801 propose plusieurs variations.

L'ampleur du décor brodé est en revanche exceptionnelle. Elle correspond à ce que Boulard fit réaliser en 1808 pour la grande chambre de l'Impératrice au rez-de-chaussée des Tuileries d'après les projets de Charles Percier (1764-1838) : une tenture en velours de soie ponceau brodé à l'or fin avec des lyres dans une couronne, des paniers de fleurs, des véla, des abeilles... dont l'organisation évoque directement les broderies du Grand Salon de Fontainebleau. Le décor des Tuileries n'est plus connu que par une aquarelle de JeanBaptiste Isabey (1767-1855) figurant Napoléon présentant le roi de Rome nouveau-né à l'impératrice Marie-Louise (New York, coll. part.) ${ }^{10}$.

11 Outre les tentures, Boulard fournit quatre paires de rideaux - en taffetas jaune à bordures brodées en rouge, agrémentées de draperies et écharpes brodées et soulignées de jasmins de soie amarante - et les couvertures semblables des sièges, dont les bois dorés sont exécutés par François-Honoré-Georges Jacob-Desmalter (1770-1841). Il s'agit des deux 
divans susmentionnés, un canapé, deux bergères, six fauteuils, vingt-six chaises, quatre tabourets de pieds et un écran de cheminée.

Dans le salon de l'Impératrice, chaque objet participe de l'harmonie rouge et jaune. Des moulures d'encadrement dorées jusqu'au marbre griotte de la cheminée et des dessus des consoles et des jardinières, en passant par les bois dorés des sièges, leurs couvertures jaune et amarante, les girandoles en bronze doré reposant sur des piédestaux en bois doré, ou encore les deux vases d'époque Consulat en porcelaine de Sèvres à fond rouge d'oxyde de fer, ceints d'un bandeau blanc ornés de médaillons à l'imitation des camées, tout s'inscrit dans cette opposition de couleurs (fig. 5). Le grand tapis livré par Bellanger (dates inconnues) en 1809, tissé d'après les dessins de Jacques-Louis de La Hamayde de Saint-Ange (dates inconnues) par la manufacture Piat Lefebvre et fils à Tournai, n’y fait pas exception.

Figure 5

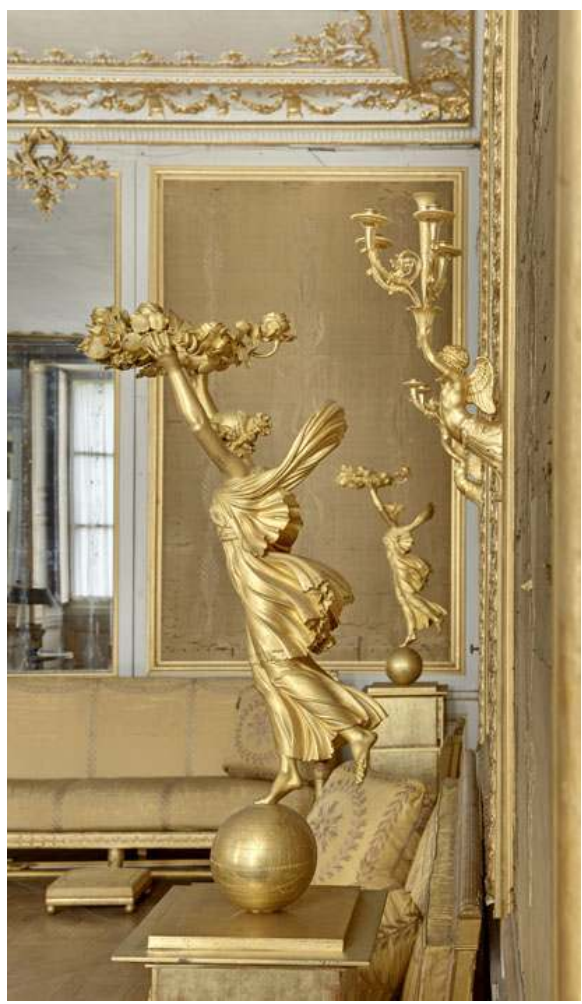

Vue du Salon jaune du côté opposé à la cheminée, état actuel. 14-542133, Fontainebleau, château. Phot. Didierjean, Adrien, 2014. (c) RMN-Grand Palais (Château de Fontainebleau).

\section{Dépose, repose, transformation, remploi}

Les travaux sont à peine achevés que Joséphine entre dans les lieux, mais elle ne profite de ses Petits Appartements que lors du séjour de 1809. Son divorce prononcé, elle cède la place à l'archiduchesse Marie-Louise de Habsbourg, la nouvelle impératrice. En prévision du séjour du couple impérial, le chiffre de Joséphine doit être supprimé. Les tapissiers Poussin et Lejeune, successeurs de Boulard, sont chargés de rebroder "en soye amaranthe sur 12 dessus d'oreiller fond jaune le chiffre ML » et de remplacer la feuille d'écran ${ }^{11}$. 
En 1814, les Petits Appartements de l'Impératrice sont mis à la disposition de la duchesse d'Angoulême, fille de Louis XVI, mariée à son cousin Charles-Antoine d'Artois, fils du futur Charles X. La pièce ne connaît pas de modification dans ses dispositions et son mobilier, mais les emblèmes et les chiffres brodés doivent être masqués. La broderie se prête difficilement à ce type d'intervention. Aussi, les tentures sont déposées en urgence avant le voyage de Fontainebleau pour faire disparaître les abeilles placées dans les mandorles des pilastres ${ }^{12}$. Avec habileté, l'intérieur des cartouches est découpé et remplacé par des pièces de taffetas jaune brodé de culots en fil de soie rouge, dont l'insertion est dissimulée par des contours brodés (fig. 6). La différence de qualité du support, du fil, de sa teinte et de la densité des points ne laisse aucun doute sur ces modifications. Sur les coussins, le chiffre J, déjà remplacé par celui de Marie-Louise, est habilement changé par des rosaces brodées (fig. 7).

Figure 6



Mandorle brodée d'un culot de fleurs, remplaçant en 1814 les abeilles brodées en 1809.

PHOT. STROUK, AgATHE, 2014. @ A AgATHE StroUK. 


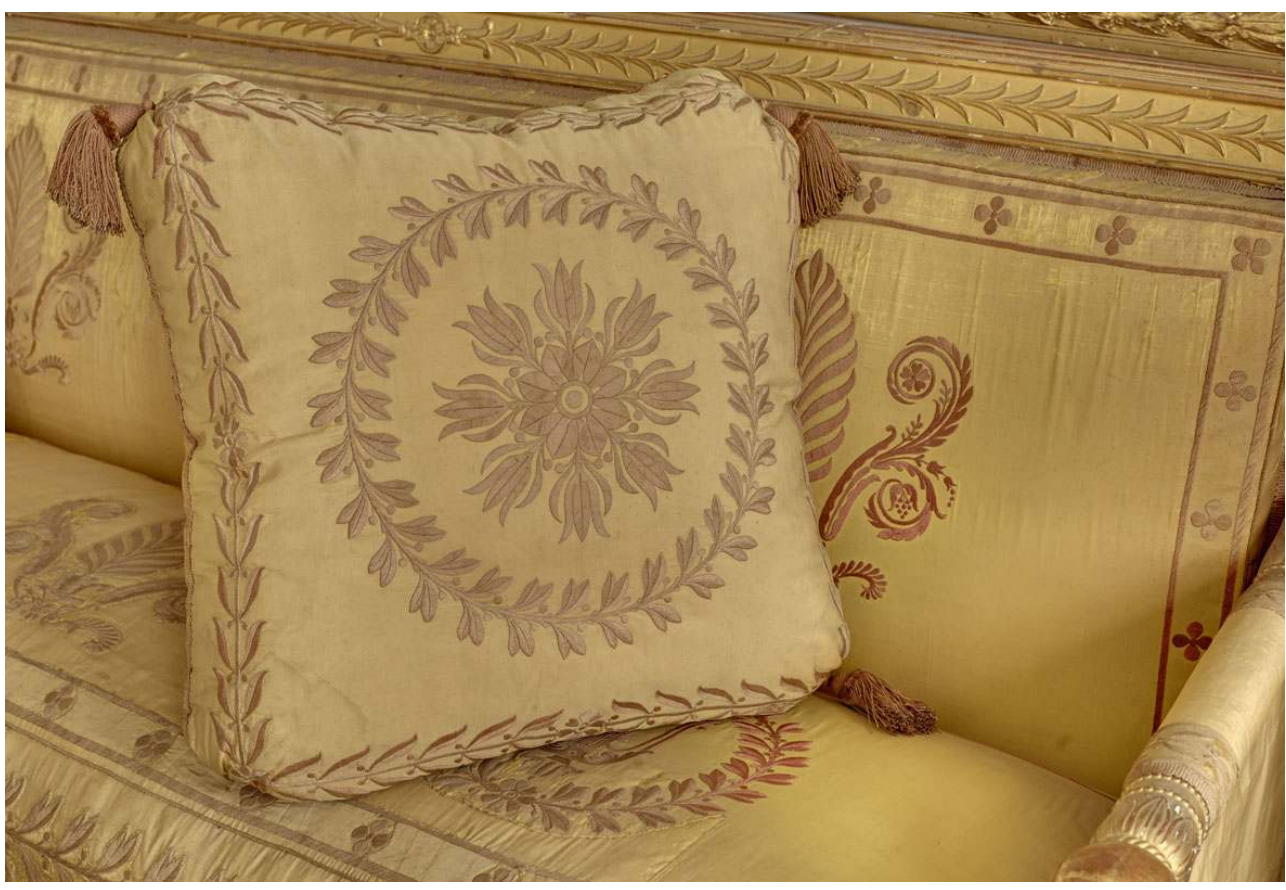

Canapé avec un de ses coussins dont les broderies ont été remplacées en 1814. 14-542134, Fontainebleau, château.

Phot. Didierjean, Adrien, 2014. (c) RMN-Grand Palais (Château de Fontainebleau).

Destiné aux princesses Marie et Clémentine, filles de Louis-Philippe, le Salon jaune traverse la monarchie de Juillet sans dommage. En revanche, sous Napoléon III, les Petits Appartements sont transformés en trois logements pour les invités de marque. Le premier (appartement $\mathrm{n}^{\circ}$ 258) comprend l'antichambre, le passage, le premier et le deuxième salon de Joséphine, ainsi que la pièce contiguë, ancien salon d'attente des dames d'honneur, transformé en chambre à coucher.

Le décor du Grand Salon est alors considérablement modifié : le plafond ancien est démoli et refait au plâtre, le conduit de cheminée est élargi et un chambranle ancien de marbre griotte remplace celui du Premier Empire, les lambris anciens sont déposés pour être remplacés ${ }^{13}$. À cette occasion, le compartimentage des lambris, qui guidait l'implantation des panneaux de tenture, est modifié. Il s'agissait d'intégrer sur le grand mur, face aux baies, des trumeaux de miroirs formant vis-à-vis avec ceux des entre-fenêtres. Ceux-ci sont alors enrichis d'ornements en carton-pierre réalisés par les frères Huber qui décorent aussi le plafond, et les dessus-de-porte, rehaussés de dorure ${ }^{14}$ (fig. 8). 
Figure 8

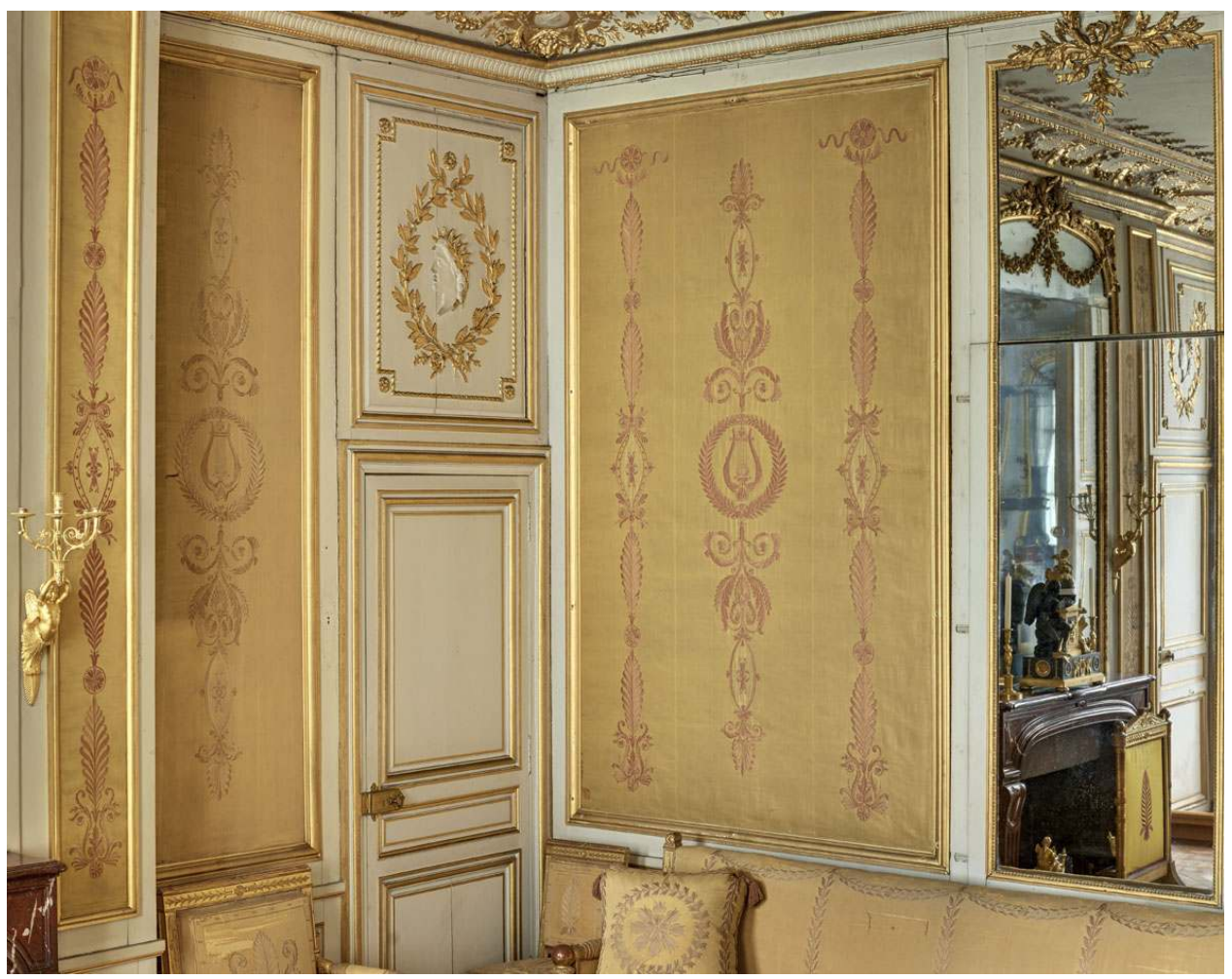

Deuxième panneau traité, vue d'ensemble. 14-542137, Fontainebleau, château.

Phot. Didierjean, Adrien, 2014. (C) RMN-Grand Palais (Château de Fontainebleau).

Les broderies de Boulard sont préalablement déposées et remplacées par « une tenture en gourgouran vert choux, doublée en toile forte, composée de 4 parties, 3 lés chacune, hauteur 2,90. ", d'où l'appellation de Salon vert ${ }^{15}$. Des rideaux en gourgouran vert sont substitués à ceux de Joséphine. Toutefois, la majorité du mobilier livré en 1809 est conservée dans le salon, avec ses couvertures de taffetas jaune brodées de rouge. Dix chaises rentrent en magasin en 1869, ainsi qu'un tabouret de pied en 1886.

Néanmoins, la tenture "en gros de Naples jaune en huit parties, 8 lés et 16 portions ${ }^{16}$ " fournie par Boulard est conservée au château jusqu'en 1865. Expédiée ensuite au Gardemeuble, elle y est transformée et renvoyée à Fontainebleau le 27 mars 1866 pour être installée dans la chambre à coucher occupant l'angle entre la cour de la Fontaine et le jardin anglais, au deuxième étage du Gros Pavillon (appartement $\left.n^{0} 363\right)^{17}$. De cette époque datent des élargissements en taffetas jaune, destinés à former " 16 pilastres en gros de Naples [...] avec plis » et l'ajout d'un encadrement formé d'un "câblé en soie cramoisi et jaune ».

\section{Le retour des broderies de Joséphine}

En 1902, les recherches menées par le brigadier Vincent sur les aménagements des Petits Appartements sous le Premier Empire incitent le conservateur du château de Fontainebleau à demander au ministre de l'Instruction publique «l'autorisation de faire déplacer, dans le salon de réception des Petits Appartements [...], les tentures en gourgouran vert et d'y substituer la tenture en gros de Naples jaune qui garnit 
actuellement une chambre du deuxième étage du Gros Pavillon. Cette mesure aurait pour effet de rétablir les choses en leur état primitif et de restituer au salon de réception sa physionomie originelle [...]. Les sièges (fauteuils, chaises, canapés) qui encore actuellement garnissent le salon de réception sont ceux-là mêmes qui furent exécutés sur l'ordre de Napoléon ${ }^{\mathrm{er}}$ pour le meubler. Ils sont recouverts du même tissu que la tenture [...]. Le tout forme un ensemble logique, parfaitement assorti et qu'il est rationnel de restituer dans son état primitif [...] ${ }^{18} »$. La démarche est louable, mais l'illusion grande de vouloir rendre au salon sa "physionomie originelle» au regard des transformations radicales du décor. L'administration donne son accord en décembre 1902 et l'architecte du palais est alors prié de faire déposer les encadrements dorés des tentures ${ }^{19}$. Dès les premiers mois de 1903, le gourgouran vert posé sous le Second Empire est déposé pour être réutilisé en 1906 dans une pièce des Petits Appartements de l'Empereur, dont l'histoire du décor est similaire à celle du Salon jaune: le cabinet topographique. La tenture en taffetas jaune de Boulard quitte les murs du deuxième étage du Gros Pavillon pour retrouver le Grand Salon de l'Impératrice. Ce retour aux sources n'offre cependant aucune conformité à l'état du Premier Empire, car les dispositions de 1859 interdisent d'y placer l'intégralité des panneaux anciens et les broderies retendues sur les murs voisinent avec une exubérante décoration blanc et or de style Louis XVI, sans lien avec les surfaces simplement moulurées de l'état de 1808-1809 (fig. 9). Par ailleurs, quelques éléments brodés sont placés en réserve, notamment un grand panneau à dessin de velum et de panier fleuri ${ }^{20}$. Le décor déraciné devient inapproprié dans le salon pour lequel il fut réalisé cent ans auparavant.

Figure 9

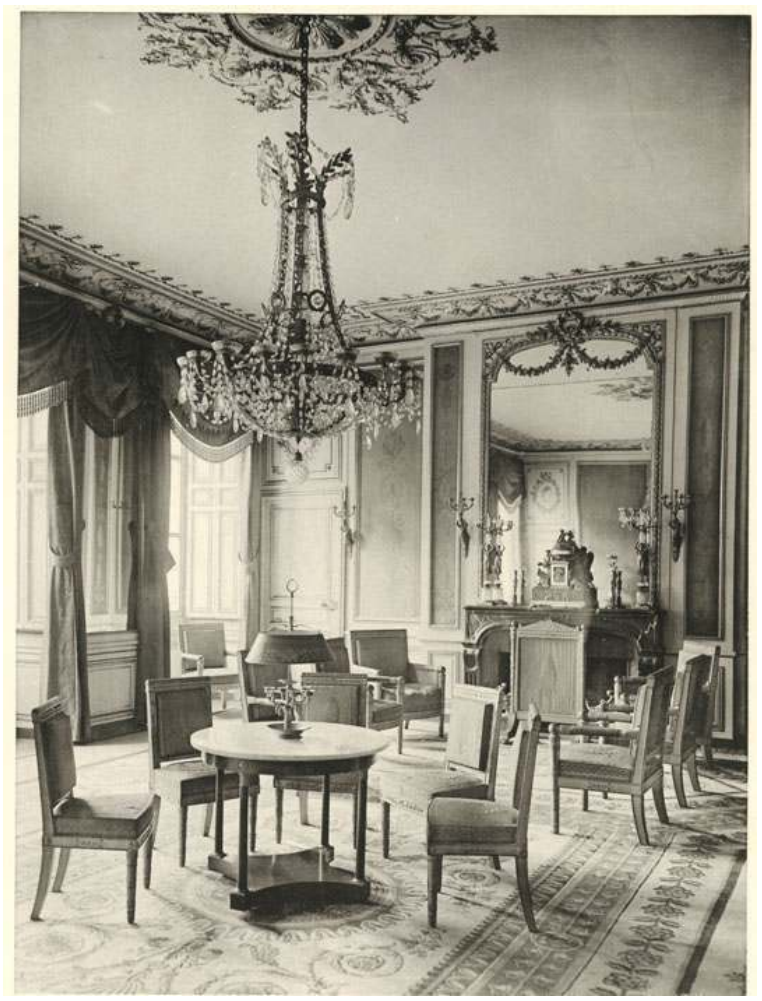

Vue du Salon jaune vers 1910 dans Georges d'Esparbès. Fontainebleau, les Petits Appartements de Napoléon et Joséphine. Versailles : Bourdier, s.d., pl. 126.

Repro. Cochet, Vincent, 2015. (C) Vincent Cochet. 
Sa réintégration dans le Grand Salon de l'Impératrice conduit cependant la conservation du château à multiplier les efforts pour accentuer la présence du décor du Premier Empire. Les pièces de mobilier, employées dans d'autres espaces du palais - notamment la grande console en bois doré, les deux jardinières et les grands candélabres de Thomire, à figures féminines en bronze doré portant des couronnes de fleurs et placées sur des piédestaux en bois doré - réintègrent le Salon et côtoient de nouveau les sièges dont les couvertures jaunes brodées de rouge sont conservées. En 1907, ces dernières font l'objet de consolidations et de reprises des broderies. En même temps, une restitution des rideaux est engagée. Mais les « encadrements brodés et appliqués ", mentionnés sous le Premier Empire, ne sont pas réalisés en broderie. Le dessin est traduit en tissage (Fontainebleau, inv. F 2440 C). Par ailleurs, les rideaux modernes n'ont pas l'ampleur indiquée sous le Premier Empire ; il s'agit de simples pentes alors que les rideaux livrés en 1809 sont des rideaux croisés. Installés dans le Salon, ils sont complétés par les draperies anciennes formant festons sur toute la façade des baies et des entre-fenêtres (Fontainebleau, inv. F 7108) (fig. 10).

Figure 10

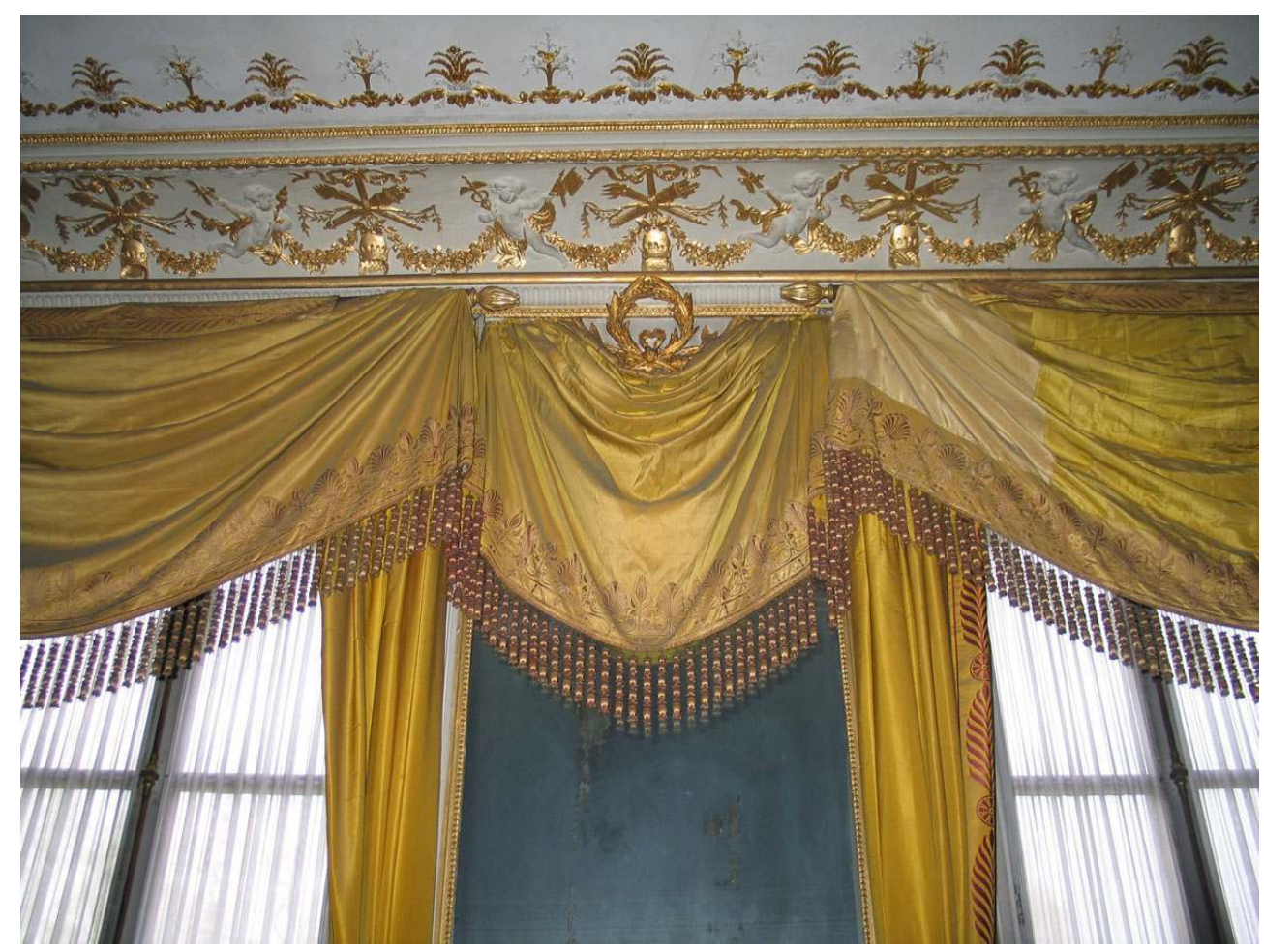

Rideaux retissés en 1907 et draperies anciennes. Fontainebleau, château.

Phot. Cochet, Vincent, 2008. (c) Vincent Cochet.

\section{Fraîcheur ou authenticité ?}

21 Ces réalisations du début $\mathrm{du} \mathrm{xx}^{\mathrm{e}}$ siècle reposent sur deux principes. Le premier est la permanence du mobilier ancien dans le Grand Salon de Joséphine depuis sa livraison en 1809 ; la volonté de l'associer de nouveau avec la tenture brodée, exilée dans des espaces non ouverts au public, offrait l'avantage de réunir un «meuble » complet, c'est-à-dire 
rendre aux tissus la place qu'ils occupaient dans le décor du Premier Empire. Le second est la volonté d'évoquer l'ameublement du Premier Empire dans l'ensemble des Petits Appartements, comme pendant aux Grands Appartements. Ces derniers ont fait l'objet d'un exercice de renforcement de leur état napoléonien pendant tout le $\mathrm{xx}^{\mathrm{e}}$ siècle. Si les décors du Second Empire sont conservés, leur présence est atténuée par la prégnance des objets du début du xix ${ }^{e}$ siècle. Au nom d'une cohérence de la présentation des lieux, le château devenu musée a fabriqué un hybride des deux Empires.

Dans le Salon jaune, la remise en place des objets et des étoffes s'est prolongée par un projet plus ambitieux. En 1970, l'idée de substituer des tissus "à l'identique» aux couvertures des sièges et aux tentures anciennes, particulièrement altérées par une exposition à la lumière, voit le jour. Les teintes du Premier Empire, particulièrement soutenues, ont en grande partie disparu pour se fondre en un camaïeu mordoré et les étoffes présentent d'importantes zones de fragilité, voire des ruptures et des lacunes.

Des essais de broderie sont réalisés en 1971, avant d'étendre la commande à la reproduction des couvertures des sièges. Toutefois, aucune des broderies neuves n'est posée sur le mobilier ancien, à l'exception de la feuille de l'écran de cheminée en 1976, dont l'original est conservé. Non abouti, ce projet de rendre au Salon la fraîcheur et l'éclat des soieries du Premier Empire consistait purement et simplement en la dépose de tous les éléments anciens pour leur substituer des copies. Ces dernières auraient nécessairement engendré la transformation du mobilier. Isolé, l'écran habillé de son étoffe aux coloris saturés possède une évidente fonction pédagogique pour évoquer l'intensité des coloris de l'ameublement du Premier Empire. Il n'en génère pas moins un déséquilibre chromatique dans la pièce (fig. 11). Le respect de l'authenticité des textiles et la restitution d'un état historique « coloré » semblent inconciliables. 
Figure 11

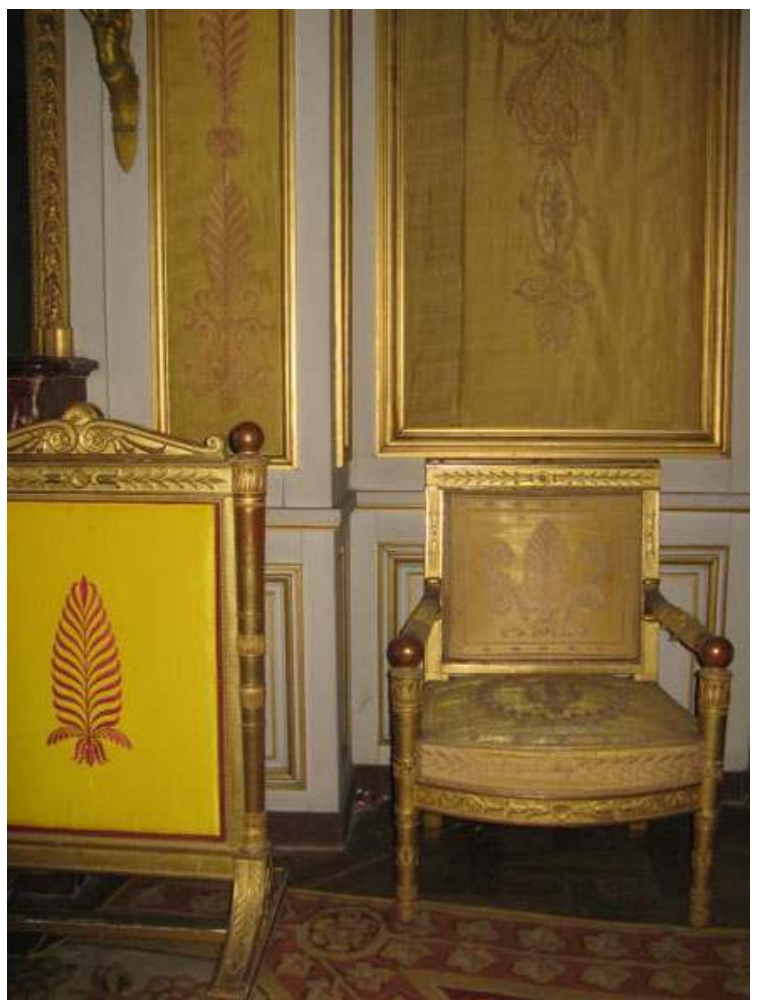

Écran de cheminée avec ses étoffes remplacées en 1976.

Phot. Cochet, Vincent, 2014. (c) Vincent Cochet. coloris frais en substituant des étoffes neuves aux tissus anciens est un débat qui est au cœur des réflexions dans les châteaux-musées. $\mathrm{Au} \mathrm{Xx}^{\mathrm{e}}$ siècle, le sacrifice des textiles d'ameublement anciens a formé un principe majoritairement admis dans le cadre de la rénovation-restitution des appartements royaux et impériaux. Les substitutions par des tissages modernes s'avèrent incontournables dans certains cas, mais le procédé n'aurait-il pas dépassé ses objectifs ? De l'idée, à la formule... à la facilité. Mais pour quel bénéfice patrimonial?

Depuis 1907, les rideaux du Grand Salon ont subi les effets de la lumière naturelle. Les tentures plus encore. Face à ces altérations, la question de la sauvegarde ou du remplacement des textiles se pose dans les mêmes termes que précédemment. Néanmoins, l'idée de concilier la préservation des éléments originaux et la restitution des coloris a entraîné la recherche de solutions de traitement novatrices.

du respect de l'authenticité de l'un des plus vastes ensembles brodés du Premier Empire encore en place répond aux missions de conservation, mais permettre de retrouver les contrastes colorés entre les fonds jaunes et les motifs rouges s'avérait un enjeu de compréhension de l'ameublement de Joséphine.

faisabilité d'une telle opération méritait d'être confirmée par une étude. À ce titre, la collecte de données techniques et l'établissement d'un constat d'état de l'ensemble ont été menés début 2013 afin de dégager des préconisations de traitement. Parallèlement, un premier essai grandeur nature a été effectué sur l'un des pilastres brodés de la cheminée 
dans le but d'évaluer les possibilités techniques de restauration et d'en apprécier le résultat esthétique.

L'enjeu d'une intervention de restauration dans le Salon jaune est de parvenir à agir à la fois sur la faiblesse structurelle des étoffes et sur la perception générale de la tenture. En effet, l'état de dégradation des douze panneaux la composant ne permet plus d'apprécier l'intention originelle du décor, notamment en raison de la perte quasi totale du contraste coloré entre le fond et les motifs brodés.

\section{Une tenture dénaturée}

La grande fragilité de la tenture est liée à l'effet combiné de la lumière, de la poussière, de la tension et de ses remaniements successifs.

L'exposition prolongée à la lumière naturelle a provoqué le virage irréversible des coloris en une teinte beige uniforme, du fait de la dégradation des colorants employés pour le tissu et les fils de broderie (fig. 12).

\section{Figure 12}

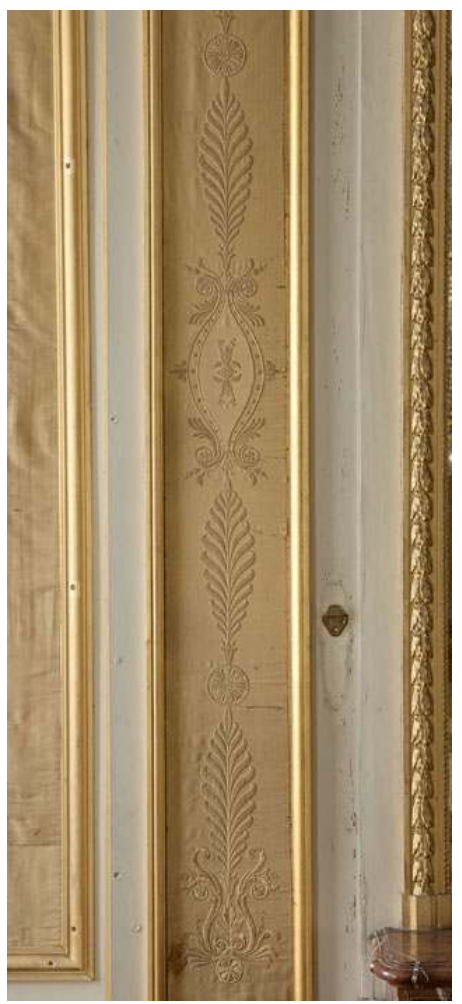

Pilastre brodé situé à gauche de la cheminée, avant restauration. 14-542138, Fontainebleau, château. Phot. Didierjean, Adrien, 2014. (C) RMN-Grand Palais (Château de Fontainebleau).

31 Une observation de l'envers des étoffes et des bordures, ainsi que des parties protégées par les coussins des canapés, permet d'avoir un aperçu des coloris et du contraste d'origine (fig. 13). En outre, les rayonnements ultraviolets et infrarouges ont entraîné des coupures dans le réseau macromoléculaire des fibres de soie, diminuant leurs propriétés mécaniques. Il en résulte une extrême fragilité aux frottements et l'apparition de nombreuses fentes, lacunes, usures et rayures, qui s'ajoutent aux accidents survenus dans 
l'histoire de la tenture. La mise en tension des étoffes favorise également l'affaiblissement des fibres et la propagation des déchirures. En toute logique, les panneaux les plus altérés sont ceux qui font face aux fenêtres.

Figure 13

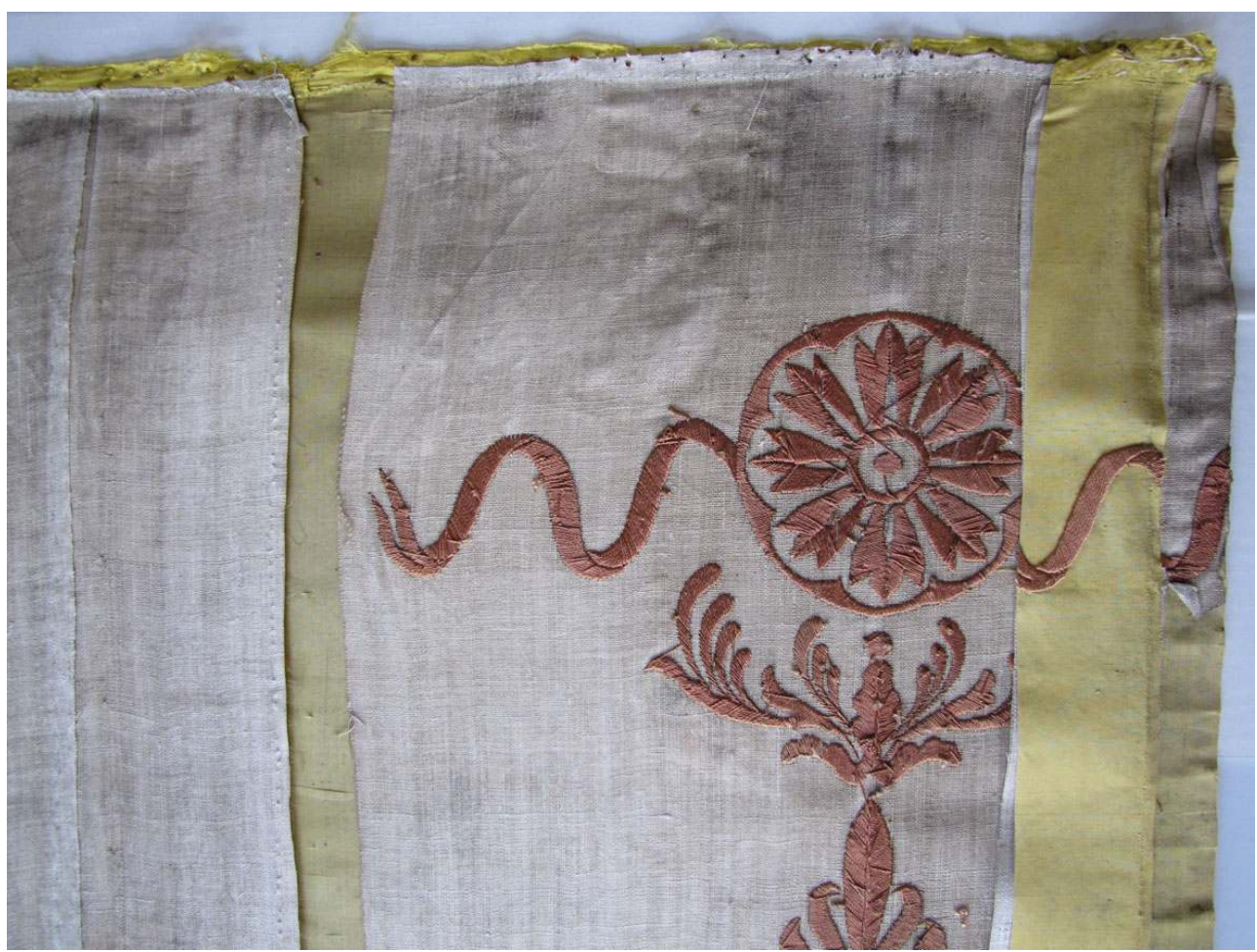

Détail du revers de l'angle supérieur gauche du deuxième grand panneau sur le mur face aux fenêtres, après dépose.

PHOT. STROUK, AgATHE, 2014. (C) AgATHE Strouk.

Par ailleurs, le montage en tambour des panneaux rend possible une circulation d'air et accélère le dépôt et l'incrustation de la poussière. La tenture agissant alors en quelque sorte comme un filtre, des zones sombres se sont formées aussi bien en surface que sur les épaisseurs sous-jacentes du montage (triplure et doublure). En outre, la poussière s'est incrustée dans les fils au niveau des dégradations de l'étoffe, soulignant ainsi fortement en noir les déchirures et les lacunes, et plus généralement, tous les accidents du tissage. L'aspect noirci et taché des panneaux brodés affaiblit donc également la perception du contraste entre le fond et les motifs (fig. 14). 
Figure 14

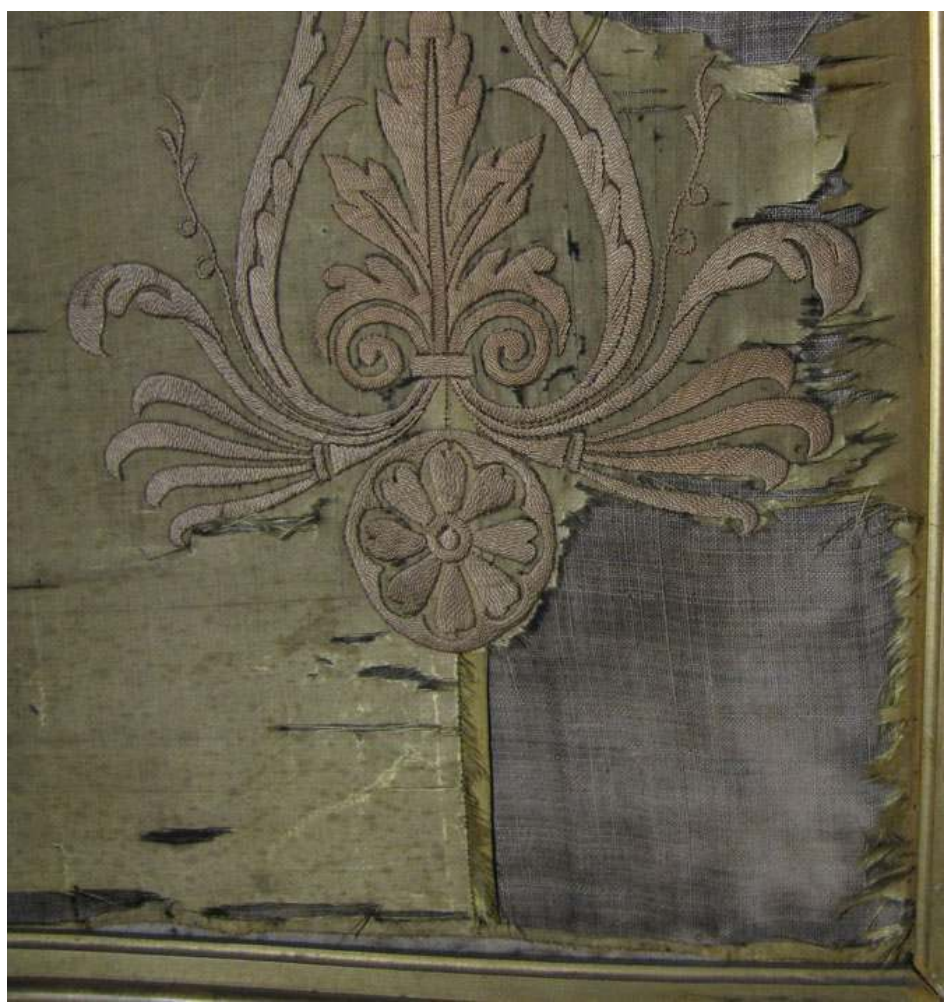

Angle inférieur du panneau de droite sur le mur face à la cheminée, avant restauration.

PHOT. STROUK, AgATHE, 2013. (c) AgATHE STROUK.

Enfin, l'histoire matérielle de la pièce explique la présence de transformations plus ou moins importantes, qui peuvent s'apparenter aujourd'hui à des dégradations: empiècements, coutures, rajouts, prélèvements, trous de clous, ajouts de galons, fantômes de motifs, reprises, ravaudages, épinglages, etc. En effet, les panneaux ont dû être tantôt raccourcis, tantôt allongés, retaillés puis élargis, réadaptés plusieurs fois au gré des réaménagements du Salon jaune ou pour s'intégrer au décor de la chambre à coucher du Gros Pavillon.

Le retour de la tenture à son emplacement d'origine a nécessité une recomposition des panneaux, certains éléments de rideaux ayant probablement été remployés pour compléter des parties manquantes ou déjà trop altérées. Ainsi, le panneau étroit tendu sur le retour de cheminée, face aux fenêtres, semble résulter de l'assemblage de deux pilastres à palmettes, cousus en leur centre. Comme sur d'autres pièces, des trous de clous de part et d'autre d'un pli témoignent d'un cloutage ancien, afin de raccourcir la hauteur (fig. 15). Les plis et les déformations visibles sur certains panneaux sont également dus à ces modifications de lieu et de destination. Les traces verticales pourraient ainsi s'expliquer par la mise en forme, sous le Second Empire, de plissés sur toute la hauteur pour former des pilastres. 
Figure 15

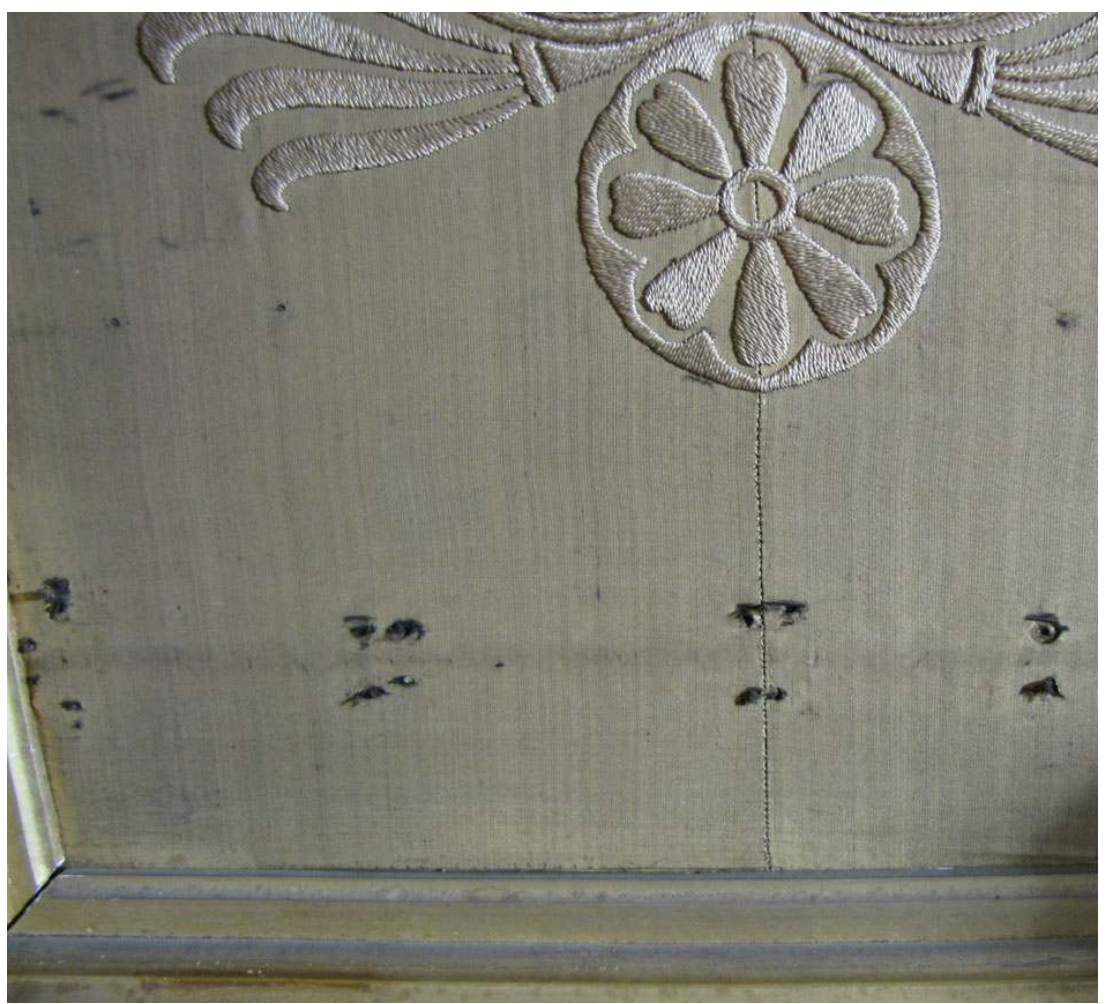

Détail du bord inférieur du panneau de gauche sur le mur face à la cheminée avec anciens trous de clous de part et d'autre d'un pli, avant restauration.

PHOT. STROUK, AgATHE, 2013. (c) AgATHE Strouk.

La recomposition de la tenture a également nécessité de dé-broder plusieurs motifs, dont le fantôme est visible sur les deux panneaux les plus larges, aux extrémités du mur face aux fenêtres (fig. 16). Ailleurs, certains motifs sont interrompus par la coupe franche des bords ou sont masqués par les boiseries, indiquant que la largeur d'origine de l'étoffe excédait l'emplacement disponible. Le panneau situé à l'extrémité droite du Salon, sur le mur faisant face à la cheminée, présente quant à lui une découpe perpendiculaire, correspondant sans doute à une ancienne ouverture de porte sous tenture (fig. 17). Les doublures elles-mêmes résultent d'un assemblage de plusieurs morceaux de lin blanc, cousus à des époques différentes et parfois superposés. 
Figure 16

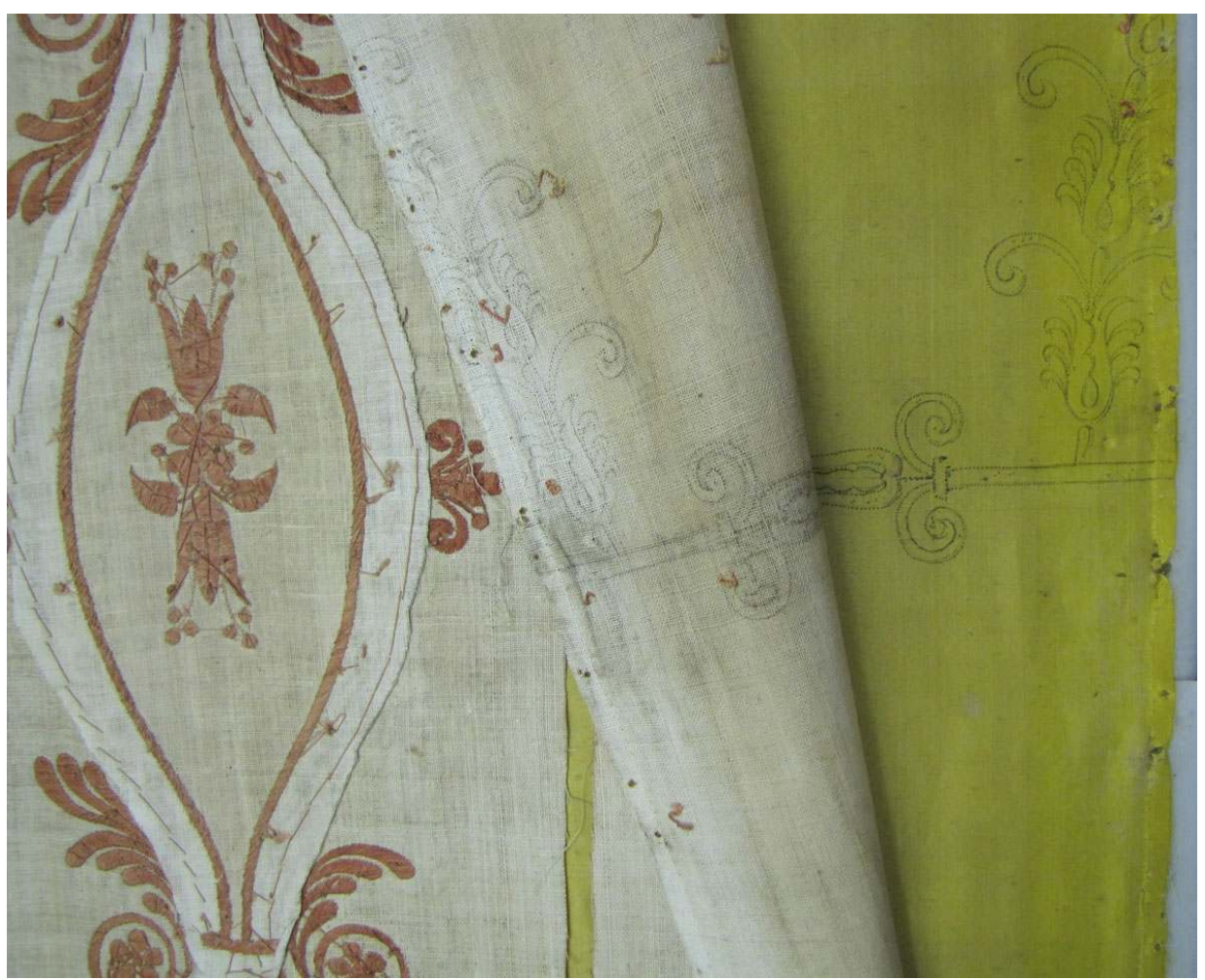

Détail du revers du panneau de gauche sur le mur face aux fenêtres en cours de démontage : fantôme d'un motif brodé et vue de la mandorle rebrodée.

PHOT. STROUK, AgATHE, 2013. (C) AgATHE STROUK. 


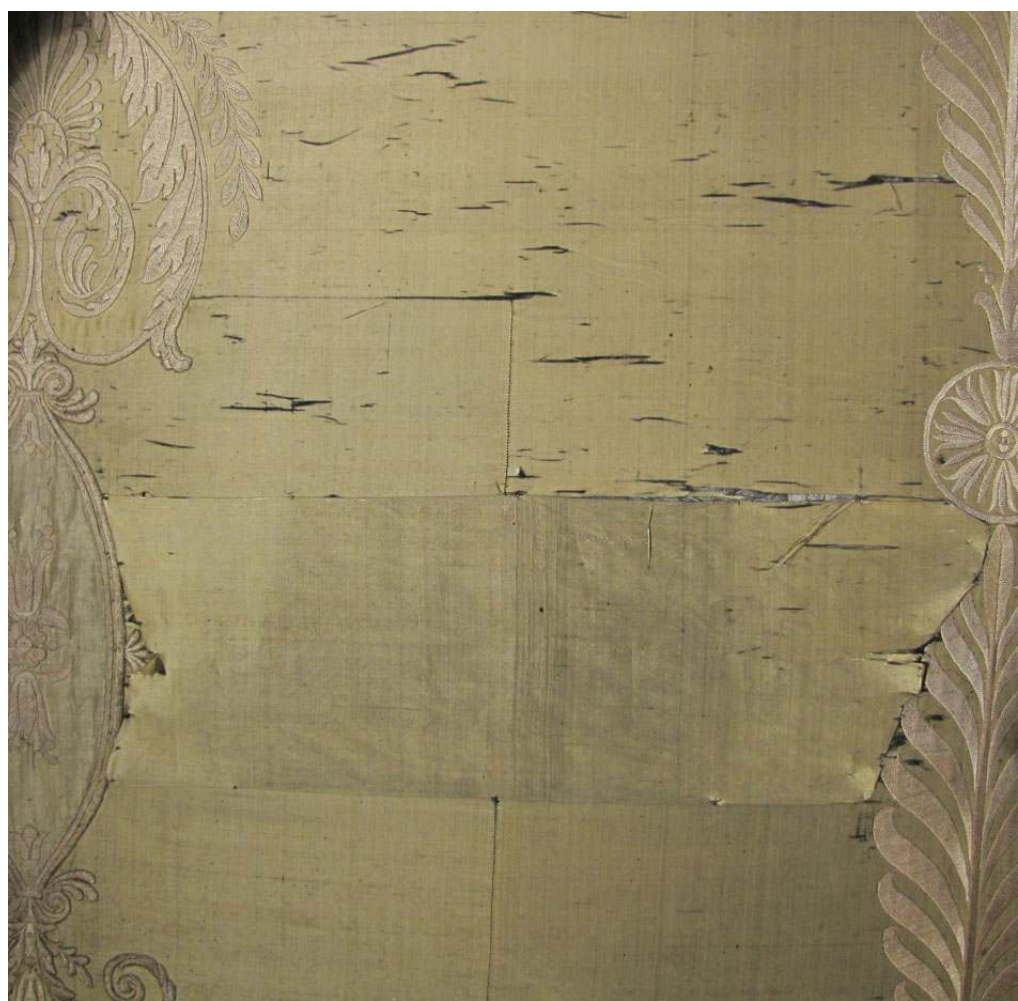

Détail du panneau de droite sur le mur face à la cheminée avec découpes et pièces d'étoffes rapportées, avant restauration.

PHOT. STROUK, AgATHE, 2013. (C) AgATHE StROUK.

À l'ensemble de ces modifications hétéroclites s'ajoutent plusieurs campagnes de réparation non datées. Dès lors, la compréhension de la disposition originale de la tenture est malaisée, d'autant que cinq éléments, conservés en réserve, n’y ont pas été réintégrés et qu'on ignore combien sont manquants.

\section{Protéger et « colorer » les textiles originaux}

La décision de maintenir en place la tenture du Salon jaune, transformée par rapport à la disposition d'origine et dénaturée par le passage du temps, a pour corollaire la restitution de sa lisibilité et de son intention décorative initiale - à défaut de son état d'origine.

Or, les contraintes du matériau textile rendent toute retouche colorée techniquement impossible et déontologiquement inacceptable : poreuse, hydrophile, non plane (du fait de l'entrecroisement des fils), la matière originale ne peut être ni recolorée ponctuellement, ni isolée par un vernis, comme on le ferait pour une couche picturale par exemple. Le geste, en plus d'être interventionniste, serait irréversible. On a donc cherché à concilier les méthodes approuvées de conservation des textiles et le réveil des coloris.

Le choix de la crêpeline de soie teinte et imprimée s'est imposé car l'utilisation de ce matériau, un voile de soie, répond à plusieurs exigences : assurer une couverture de protection assez efficace contre la poussière et les frottements, rehausser les coloris tout en laissant les matériaux visibles et perceptibles, grâce sa transparence, et enfin, pouvoir effectuer une consolidation des panneaux les plus altérés de façon efficace et réversible. 
L'adéquation des matériaux de restauration avec ceux d'origine serait également respectée. protéger par la face grâce à un voile de soie bicolore venant se superposer aux motifs originaux. Ce procédé présente l'intérêt d'éviter l'effet de voile qu'engendre souvent la pose d'une crêpeline d'une teinte uniforme sur un textile à motifs. En outre, la finesse de la crêpeline n'occulte en rien les fils de broderie et permet une parfaite lisibilité de leur relief et texture.

Le panneau situé à droite de la cheminée est choisi pour son étroitesse $(30 \mathrm{~cm}$ de large environ) et l'état relativement bon du support de soie, ce qui justifie une intervention sans aucun démontage. Un dépoussiérage approfondi au moyen d'un aspirateur de faible puissance permet l'élimination des poussières et des salissures sèches de surface, et ravive déjà légèrement les coloris.

42 échantillonnage abondant afin d'atteindre l'effet visuel recherché: il convient de déterminer la hauteur de ton du fond jaune, conjointement à la nuance de rouge et son intensité - ce qui multiplie les combinaisons possibles. L'envers d'une des bordures conservées en réserve ${ }^{21}$ nous sert de référence pour être au plus près des coloris d'origine sans les trahir. Plusieurs dizaines d'échantillons ont été testés en les plaçant directement devant l'original (fig. 18). La combinaison de coloris finalement retenue procède d'un mélange de colorants qui pourra être reproduit pour traiter le reste de la tenture.

\section{Figure 18}

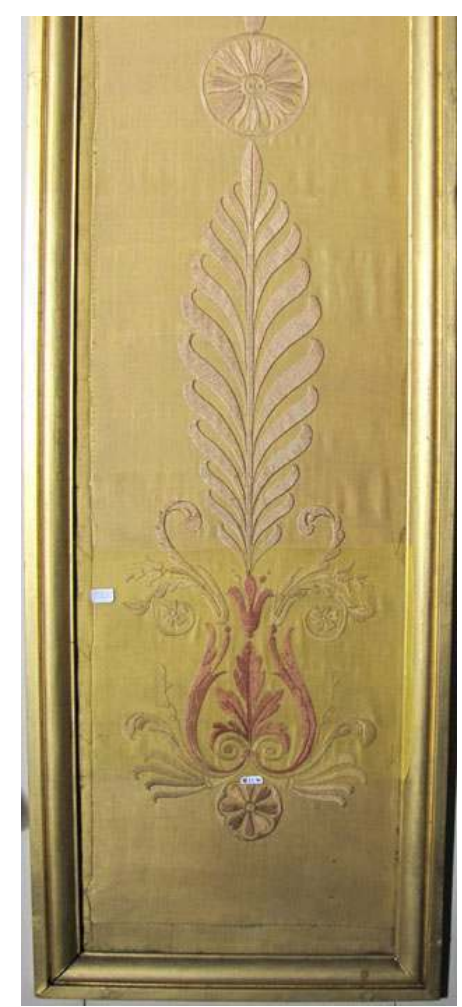

Choix d'un échantillon de crêpeline teinte et partiellement imprimée sur le pilastre brodé situé à droite de la cheminée, en cours de restauration.

PHOT. STROUK, AgATHE, 2013. @ AgATHE STROUK. 


\section{principe de la sérigraphie, est employée (fig. 19). Le séquençage des motifs est d'abord} relevé par décalque sur un film transparent, puis plaqué sur un cadre tendu d'une gaze polyester. Cette gaze a préalablement été enduite d'une émulsion photosensible durcissant au contact d'un rayonnement ultraviolet : aux endroits protégés par les traits du dessin, l'émulsion ne durcit pas. Un lavage à l'eau débouche ensuite les pores du tamis, reportant ainsi avec une grande précision, en négatif, le motif à imprimer.

Figure 19

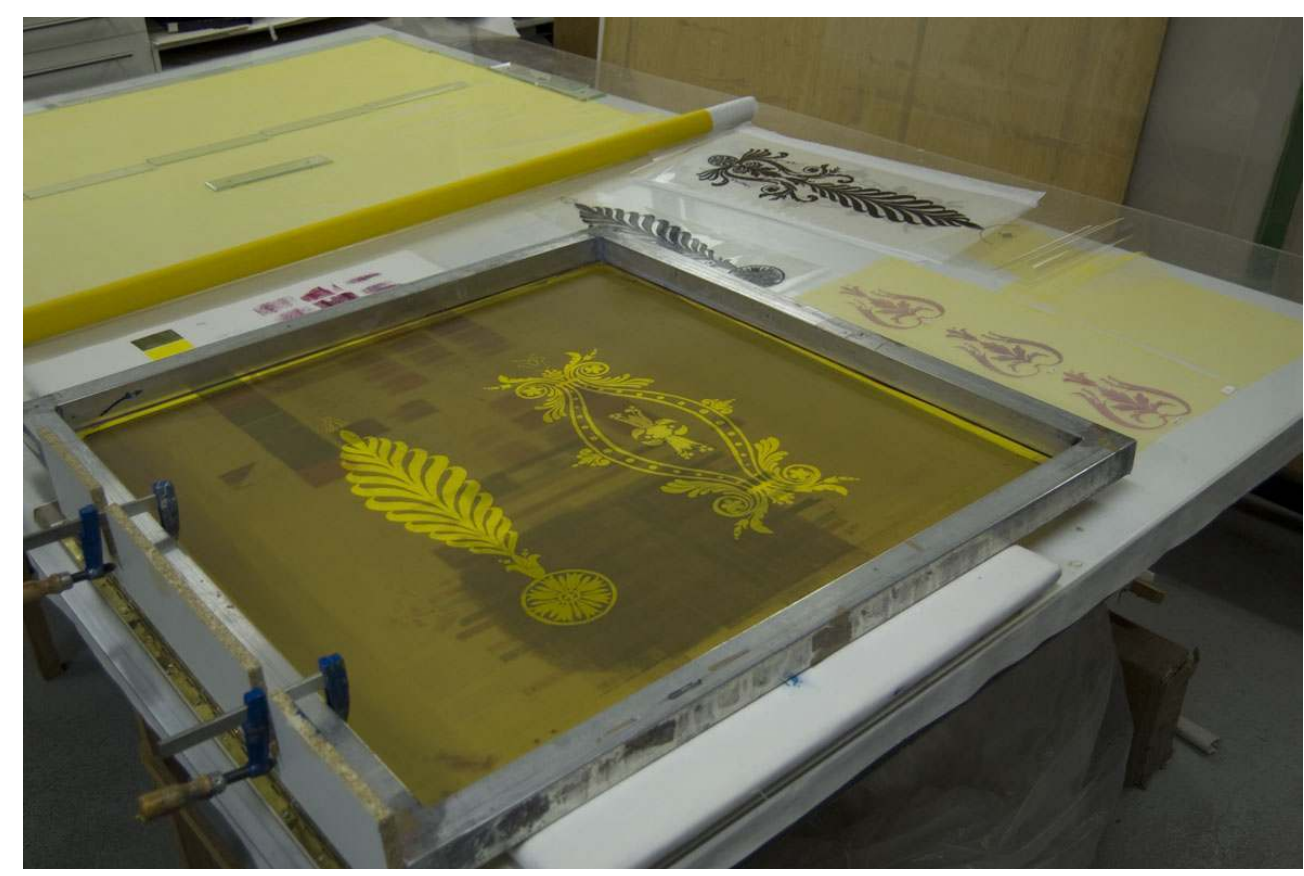

En cours d'impression de la crêpeline : cadre de sérigraphie en place, essais colorés, décalque des motifs et crêpeline prête à imprimer.

Phot. Le Namouric, Bruno, 2013. @ B Bruno Le Namouric

Pour procéder ensuite à l'impression, la crêpeline est soigneusement tendue et fixée sur la table d'impression, en veillant au droit-fil. La pâte d'impression est raclée au travers du tamis gravé, se déposant sur la crêpeline uniquement selon les motifs. L'encre d'impression se compose d'un mélange de pigments synthétiques de type acrylique, sans formaldéhyde, sous forme de dispersion ${ }^{23}$. Une fixation en étuve est alors indispensable pour garantir la stabilité des pigments dans le temps (phénomène de polymérisation sur les fibres).

La dernière étape de l'intervention consiste à fixer la crêpeline sur la tenture au moyen de points de couture prenant uniquement les broderies. En effet, les cordonnets de soie sont les seules zones résistantes, le fond jaune étant trop altéré pour supporter le passage d'une aiguille (fig. 20). 
Figure 20

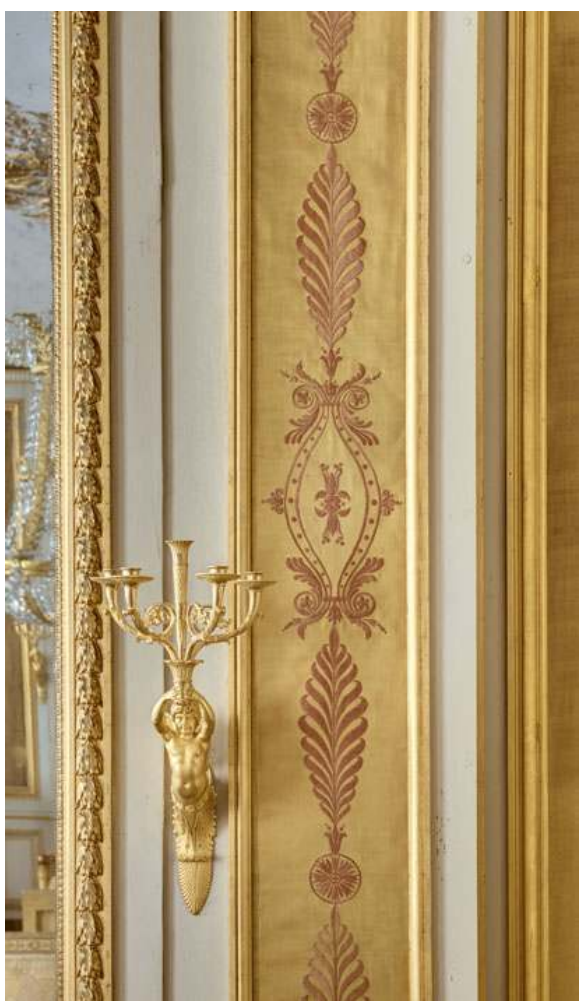

Pilastre brodé situé à droite de la cheminée, après restauration. 14-542135, Fontainebleau, château.

Phot. Didierjean, Adrien, 2014. (c) RMN-Grand Palais (Château de Fontainebleau).

\section{Traitement à grande échelle}

En raison des dimensions et de l'emplacement du panneau traité, l'étude de faisabilité s'est prolongée en traitant de manière similaire un deuxième panneau brodé qui présente des dimensions plus importantes et se situe face aux fenêtres. Celui-ci, plus altéré, reçoit directement la lumière extérieure. Il convenait donc de vérifier le faible impact visuel du doublage par la face et de juger de l'effet optique produit. De plus, de multiples problèmes techniques devaient être résolus avant de valider chaque étape du traitement. Pour réaliser l'intervention, le panneau a été déposé du mur et emporté en atelier ${ }^{24}$.

Le démontage des galons de pourtour et de la doublure en lin est indispensable pour pouvoir accéder à l'envers de l'étoffe et permettre ainsi une consolidation efficace (fig. 21). Les rangées de points de baguage fixant la doublure - probablement exécutés lors du dernier remontage des panneaux dans le Salon - sont ainsi coupés et retirés, avant même le transport de la tenture afin d'éviter les déchirures. 


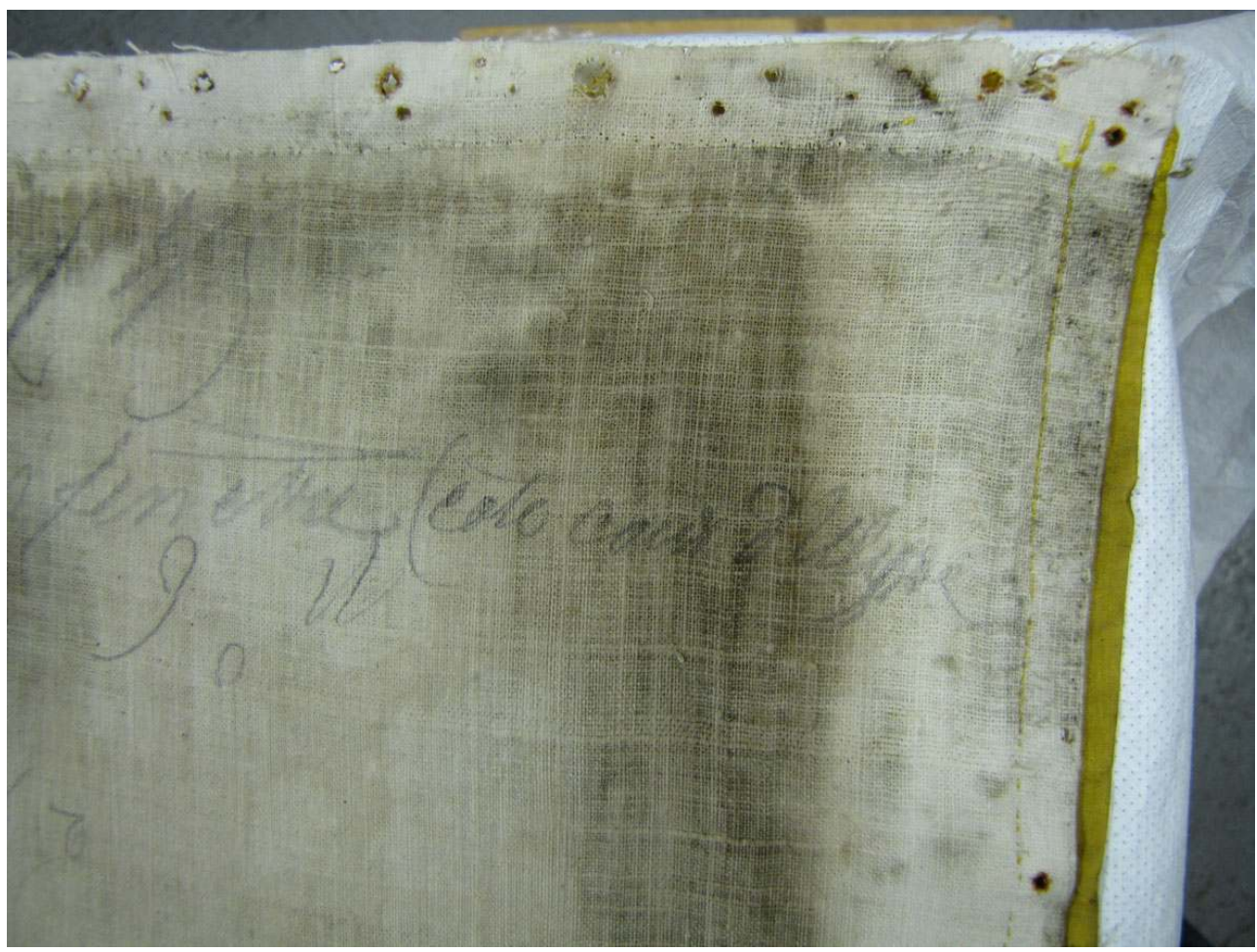

Détail du revers du deuxième panneau traité (grand panneau de gauche sur le mur face aux fenêtres) en cours de démontage des galons et de la doublure qui comporte des inscriptions anciennes à la mine graphite.

PHOT. STROUK, AgATHE, 2013. (C) AgATHE STROUK.

Dès lors, un dépoussiérage approfondi peut être mené sur toutes les couches d'étoffes, face et revers : le gros de Naples, la triplure en lin (support des borderies) et la doublure (fig. 22). Un gommage léger de toute la surface, à l'aide d'une éponge douce, permet de parfaire le dépoussiérage en atténuant certaines traces et taches (fig. 23). 
Figure 22

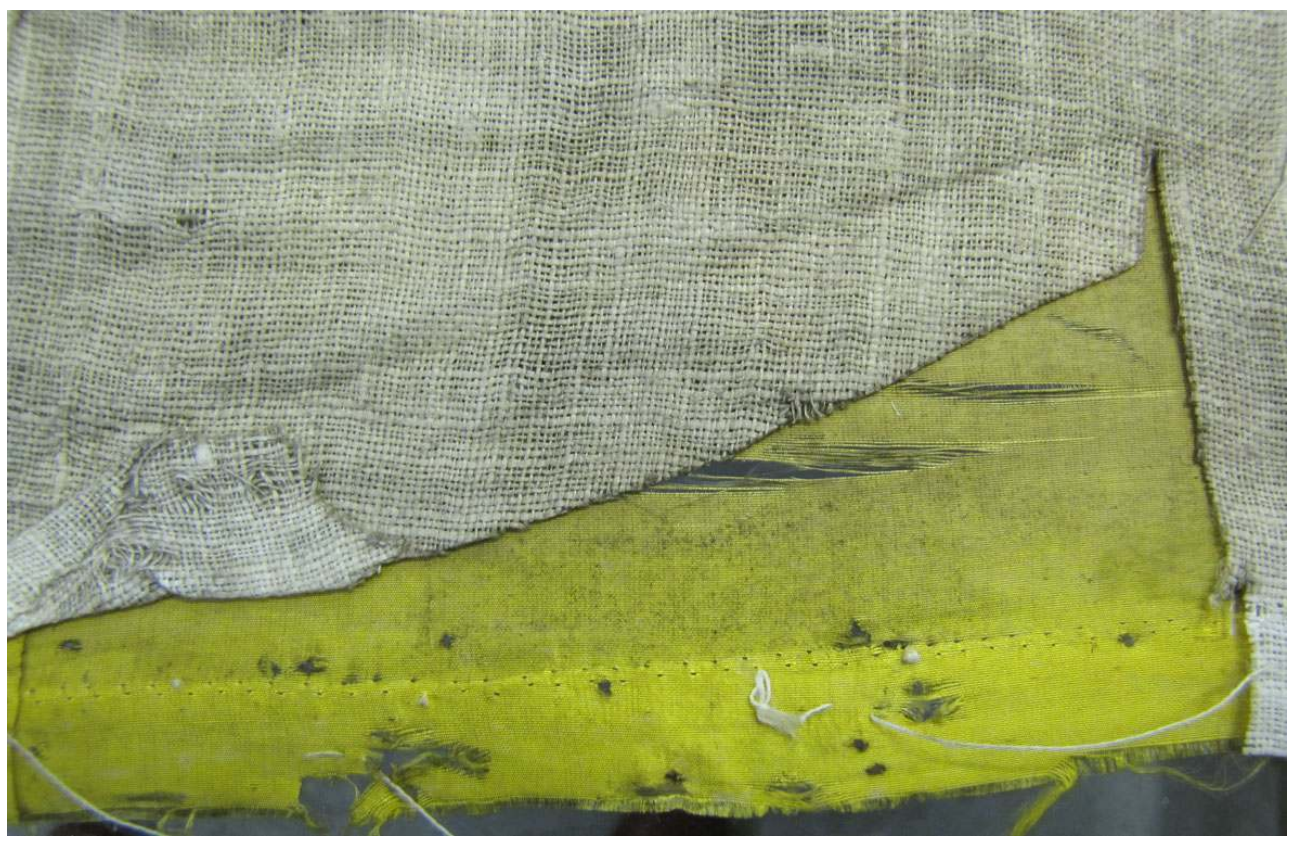

Détail du revers du deuxième panneau traité après retrait de la doublure : dépôt de poussière entre la soie et la triplure.

PHOT. STROUK, AgATHE, 2013. @ AgATHE StROUK.

\section{Figure 23}

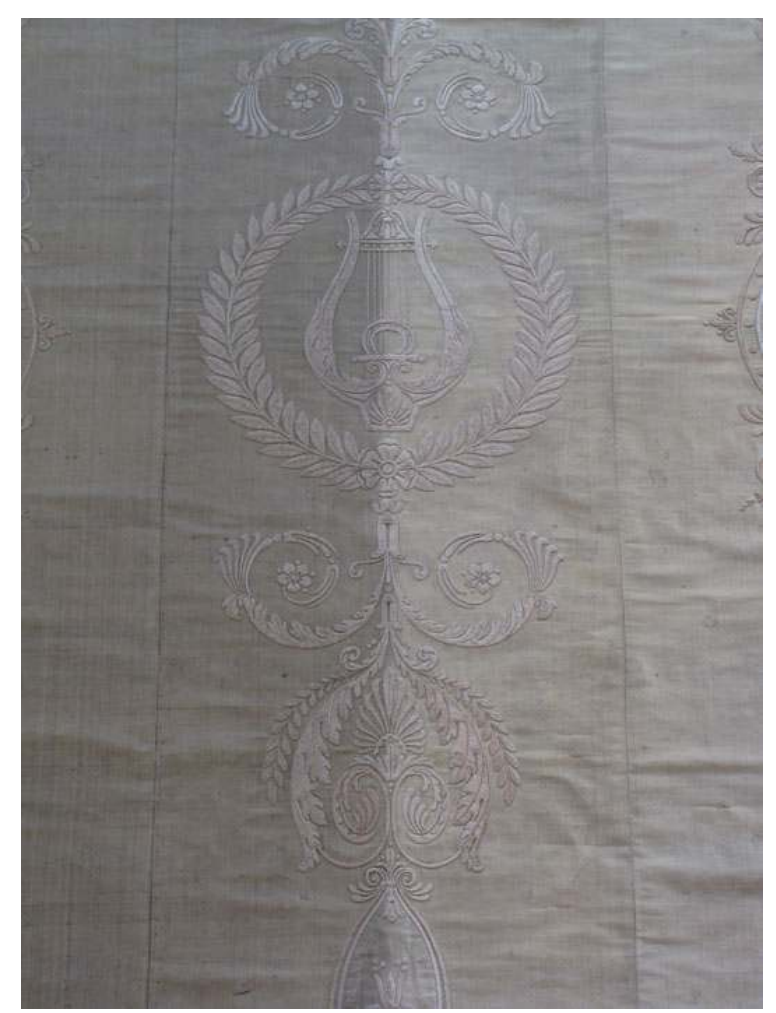

Détail du troisième panneau traité (deuxième panneau de gauche sur le mur face aux fenêtres) en cours de dépoussiérage, la moitié gauche n'ayant pas encore été traitée.

PHOT. STROUK, AgATHE, 2014. (C) AgATHE STROUK. 
La consolidation de la soierie nécessite de la doubler sur un support de pongée de soie. Teint en harmonie avec la couleur du fond, il comblera les fentes et les lacunes audessous. Or, du fait de la présence de bandes de triplure sur le pourtour des broderies au revers, il est impossible de doubler la soierie intégralement. Des pièces de pongée de restauration sont donc façonnées de manière à s'insérer au plus près des motifs brodés, entre le fond en soie et la triplure (fig. 24). Ces pièces sont ensuite glissées par le revers et maintenues uniquement par des coutures exécutées dans la triplure, toujours afin d'éviter de piquer le gros de Naples fragile.

Figure 24

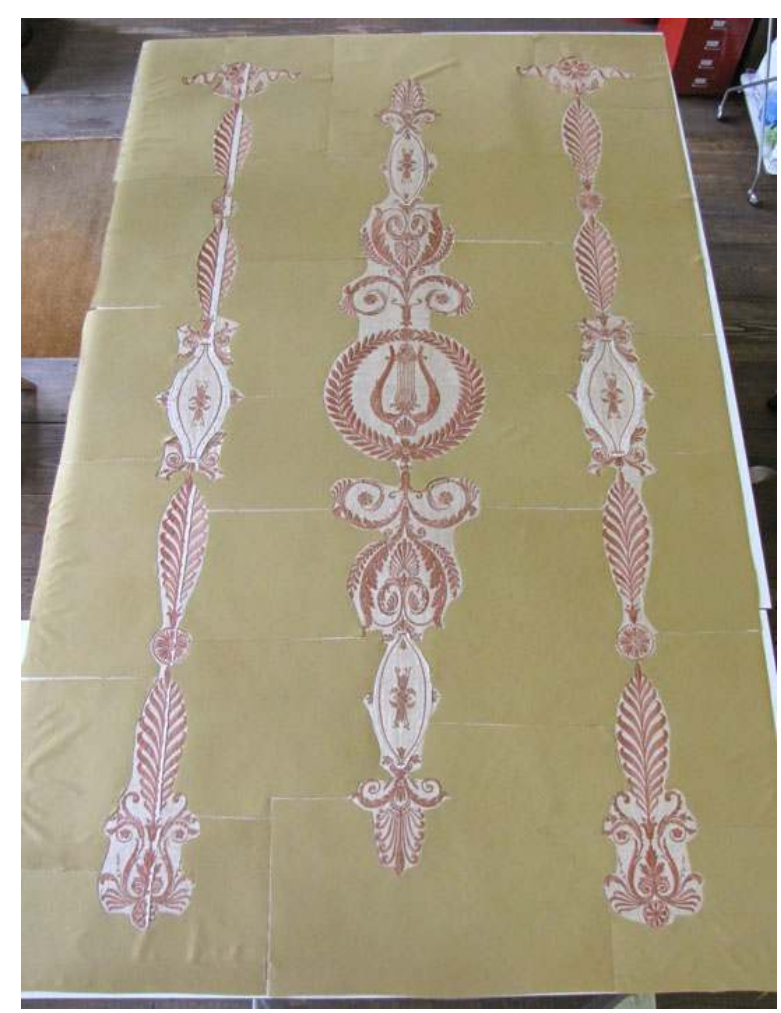

Revers du deuxième panneau traité (grand panneau de gauche sur le mur face aux fenêtres) montrant le positionnement des pièces de soie teinte avant leur introduction entre la soierie et la triplure.

PHOT. STROUK, AgATHE, 2013. (C) AgATHE STROUK.

51 Vient ensuite la préparation de la crêpeline imprimée. Comme pour le panneau-test, les motifs sont relevés précisément à échelle 1, puis noircis en positif (fig. 25). La préparation de ce décalque se fait à la main pour assurer une impression parfaitement à l'échelle et éviter ainsi tout décalage entre le motif brodé et le motif imprimé superposé, qui provoquerait une sensation de flou. 


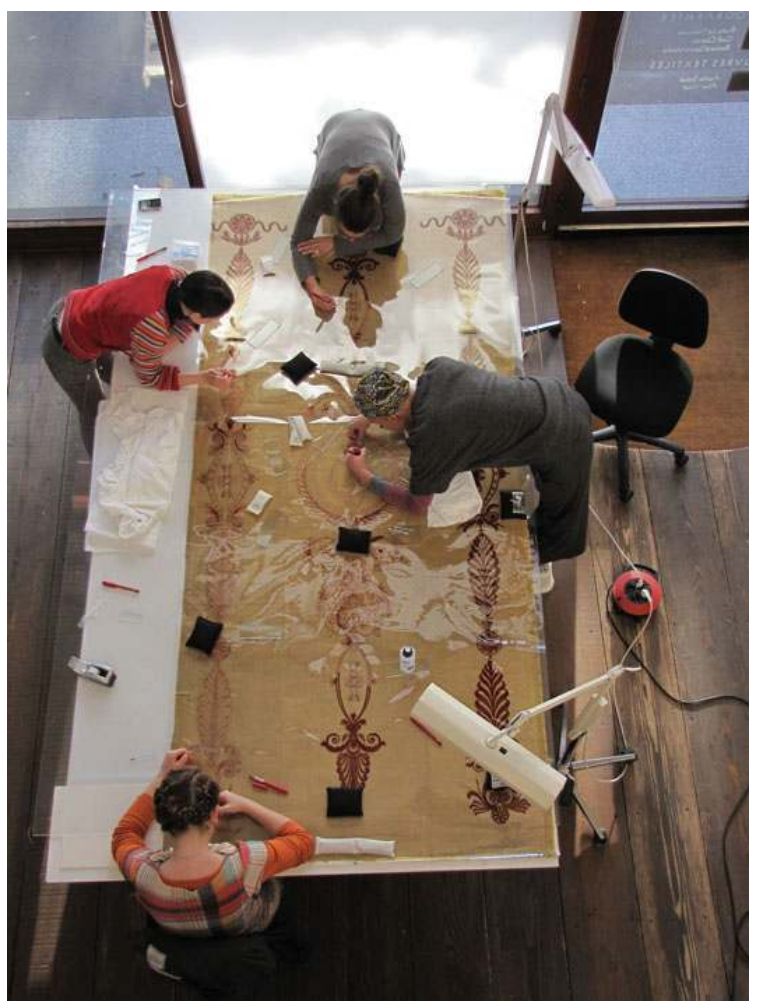

Décalque à échelle 1 du motif brodé sur le troisième panneau traité.

PHOT. STROUK, AgATHE, 2014. () AgATHE STROUK.

Le cadre de sérigraphie est préparé en atelier spécialisée ${ }^{25}$, aux dimensions du panneau de tenture. De cette manière, le motif peut être imprimé en un seul passage de raclette, ce qui garantit une plus grande uniformité de la densité de l'impression sur toute la hauteur (qui est d'environ $260 \mathrm{~cm}$ ). La largeur du panneau excédant celle de la crêpeline disponible dans le commerce ( $176 \mathrm{~cm}$ de tenture pour $140 \mathrm{~cm}$ de crêpeline), des raccords sont inévitables - ce qui ne sera pas le cas pour les tentures suivantes. Les trois séquences de motifs sont donc imprimées sur trois lés de crêpeline distincts (fig. 26, fig. 27). Ils sont ensuite fixés à la chaleur puis remis à plat à l'humidité pour s'assurer de leur concordance avec le relevé à échelle 1. 


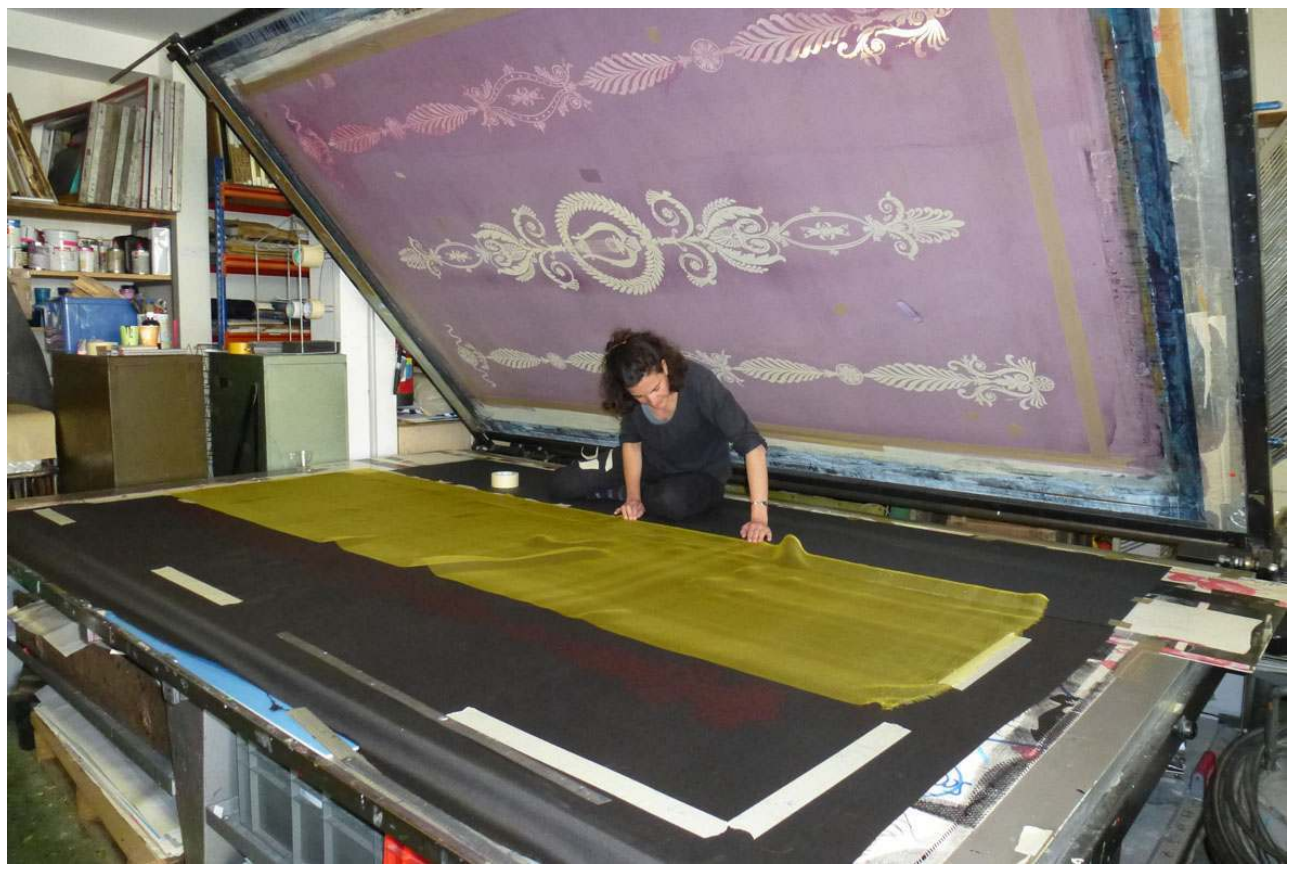

Mise en place de la crêpeline sur la table d'impression sous le cadre de sérigraphie déjà positionné. PHOT. STROUK, AgATHE, 2014. (c) AgATHE STROUK.

Figure 27

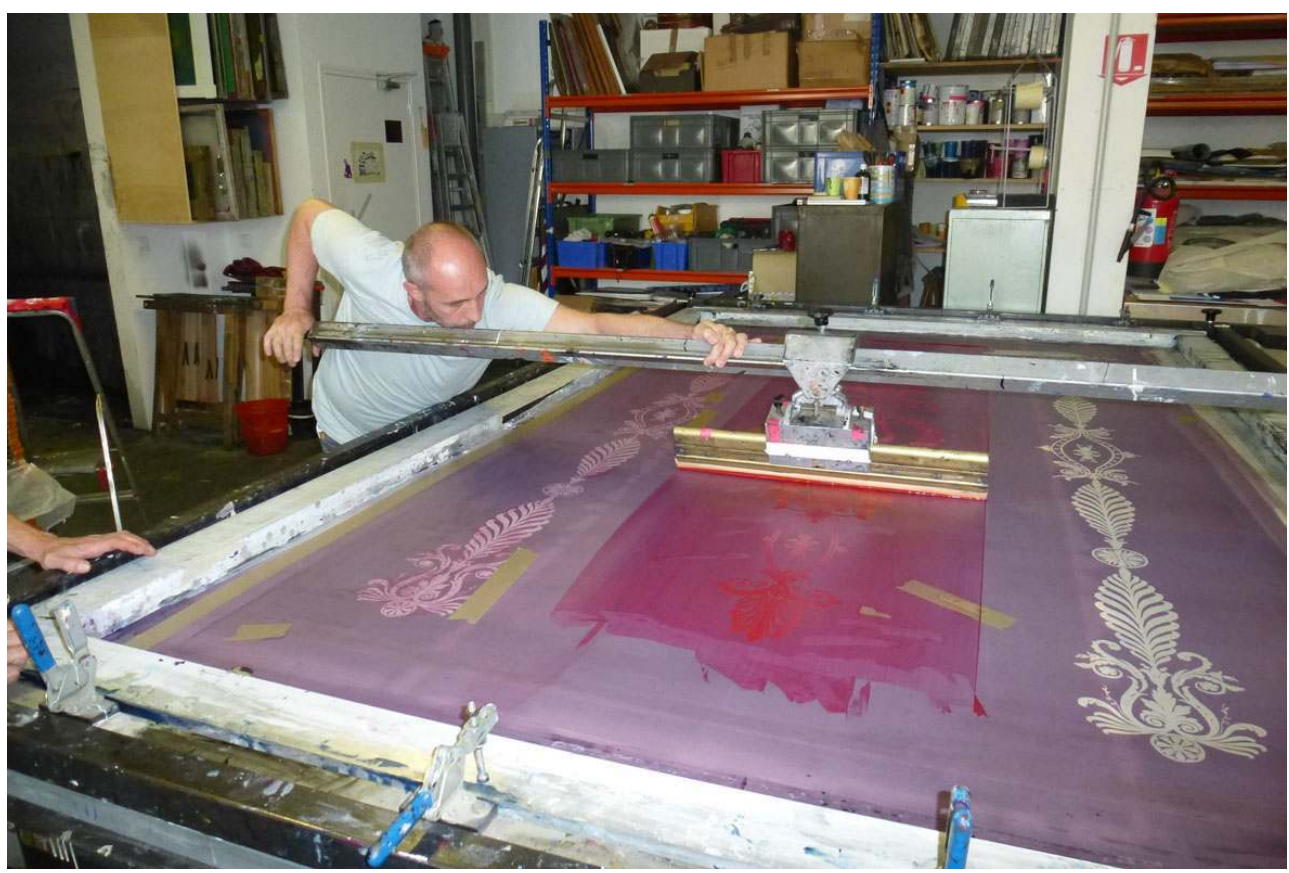

En cours d'impression du lé de crêpeline central à l'atelier EFil de Lynx, par Christophe Michel.

PHOT. STROUK, AgATHE, 2014. (C) AgATHE StROUK.

53 La fixation de la crêpeline imprimée se fait au plus près par couture au fil d'organsin de soie teint au niveau des broderies et sur le pourtour - dans la zone masquée par les boiseries, plus résistante. Plusieurs épinglages et réajustements sont nécessaires avant la 
pose définitive, pour régler les légers décalages dus à la tension (fig. 28, fig. 29). Puis, des lignes de points droits sont exécutées dans les fentes de la soie, afin de solidariser la crêpeline avec la toile de consolidation sous-jacente, sans piquer dans l'original, selon la technique dite de mise en sandwich. Les deux coutures d'assemblage des lés sont réalisées en dernier lieu, à plat au fil d'organsin sans fixer le fond en soie, en veillant à leur rectitude et à leur discrétion.

Figure 28

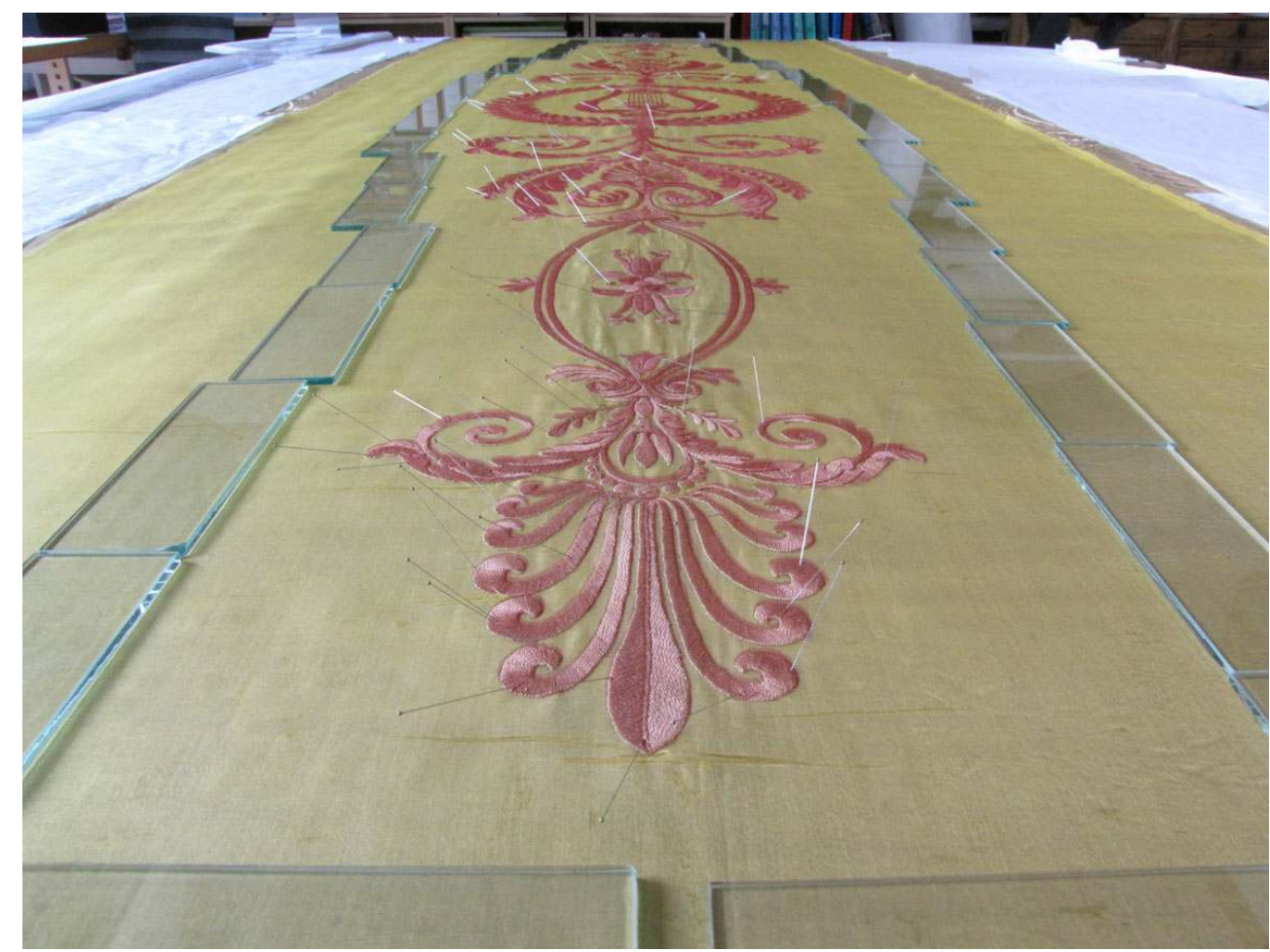

Épinglage de la crêpeline imprimée sur le motif central du deuxième panneau traité, en cours de restauration.

PHOT. STROUK, AgATHE, 2014. (C) AgATHE STROUK. 
Figure 29

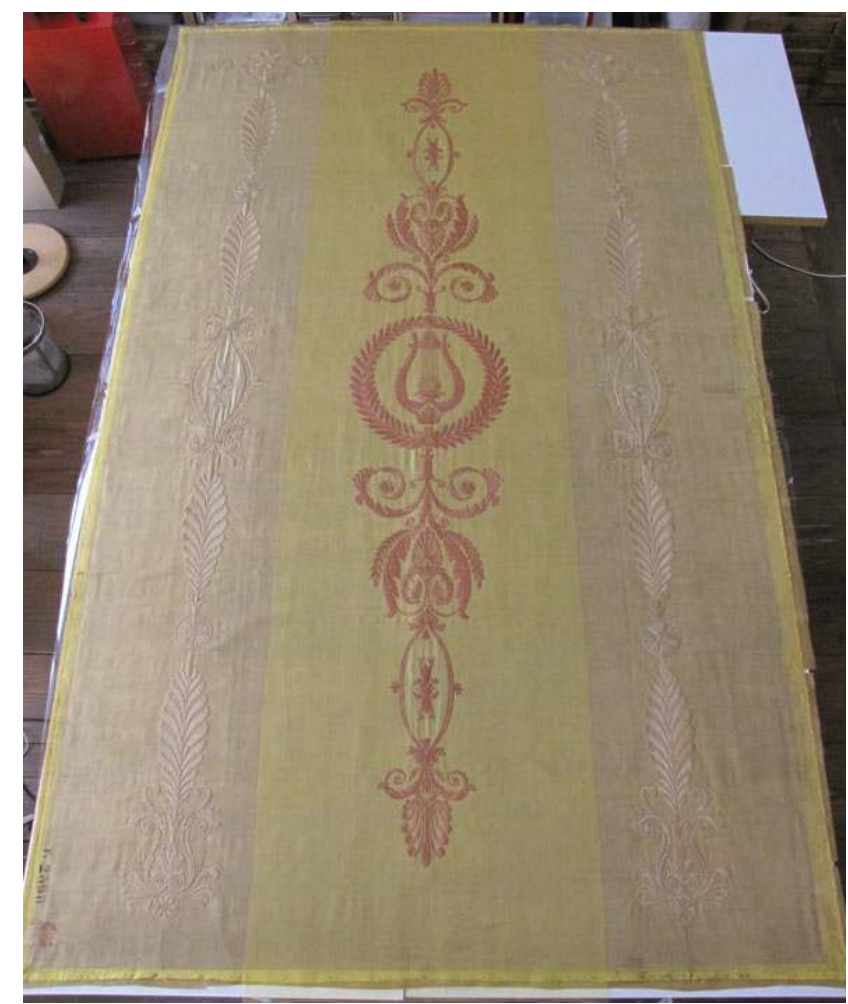

Vue générale du deuxième panneau traité après la fixation du lé central de crêpeline imprimée.

PHOT. STROUK, AgATHE, 2014. (C) AgATHE STROUK.

La doublure peut alors être reposée au revers et fixée par des lignes de points droits en quinconce, traversant uniquement les broderies et les bords. Les galons d'origine sont replacés à l'identique et cousus après y avoir introduit une bande de renfort en toile de coton, afin de combler les anciens trous de semences. De cette manière, la tenture conserve au maximum son montage ancien et l'apport des matériaux de restauration reste limité.

Avant la remise en place du panneau dans le Salon jaune, une toile de coton sans apprêt est tendue sur le mur pour assurer la propreté de la surface de repose et ajouter une épaisseur d'isolation supplémentaire contre la poussière. Enfin, le panneau restauré est refixé à son emplacement par cloutage avec des semences vernies ${ }^{26}$, en respectant la légère tension d'origine (fig. 30). 
Figure 30

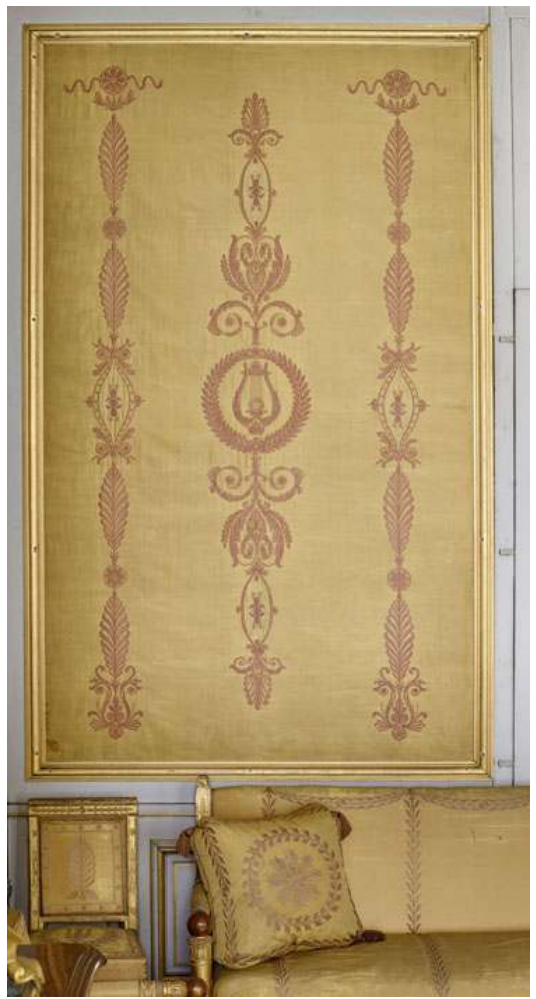

Grand panneau de gauche, sur le mur face aux fenêtres, après restauration. 14-542139, Fontainebleau, château.

Phot. Didierjean, Adrien, 2014. (c) RMN-Grand Palais (Château de Fontainebleau).

\section{Une étude concluante}

Le traitement des deux premiers panneaux du Salon jaune a donc permis de mettre au point les méthodes adéquates pour répondre aux objectifs de cette campagne de restauration. La plupart des difficultés de mise en œuvre, liées principalement au format et à la fragilité des étoffes, ont pu être réglées.

Partenaire du château de Fontainebleau, la Fondation du patrimoine a décidé de soutenir le projet. Grâce à son financement, l'intégralité de la restauration de la tenture peut être réalisée. Afin de ne pas défigurer le décor de la pièce, qui reste ouverte à la visite durant les travaux, la repose d'un panneau restauré entraîne la dépose du suivant, ce qui, panneau après panneau, correspond à un chantier de plus de trois ans qui devrait se prolonger par le traitement similaire des étoffes du mobilier; l'écran de cheminée sera prochainement doté de ses feuilles originales, doublées de crêpeline teinte et imprimée.

Bien modeste face à l'étendue des surfaces brodées à traiter, le premier panneau restauré pouvait passer inaperçu pour l'œil non-averti, plus attiré par les tons vifs de la feuille d'écran restituée en 1976 que par les demi-teintes de l'essai. L'impact de cette expérience peut être pris à mesure que la restauration progresse, notamment sur les quatre panneaux du mur situé face aux fenêtres, représentant près de $12 \mathrm{~m}^{2}$ de tissu brodé original. Cet effet est d'autant plus sensible que la pièce amorce une douce métamorphose grâce aux opérations de nettoyage des vases et du tapis, mais surtout à celles consacrées 
aux bronzes et au mobilier en bois doré livrés en 1809. L'éclat retrouvé des dorures au mercure de Thomire et surtout le traitement des bois dorés, assuré avec constance et délicatesse par les ateliers du C2RMF, démontre, s'il en est besoin, l'ampleur des efforts qu'il convient de réaliser pour transmettre aux générations futures cet ensemble qui témoigne des aménagements réalisés pour Joséphine. Réponse parfaitement adaptée aux exigences énoncées pour la tenture et le mobilier du salon jaune, cette opération méticuleuse, légère et réversible ouvre des perspectives pour le traitement en conservation des textiles anciens.

\section{BIBLIOGRAPHIE}

Sites :

La tribune de l'art : http://www.latribunedelart.com/a-fontainebleau-une-techniquerevolutionnaire-de-restauration-des-tissus

La Fondation du patrimoine : https://www.fondation-patrimoine.org/fr/ile-de-france-12/tousles-projets-593/detail-salon-d-attente-des-dames-d-honneur-de-l-imperatrice-15640R\%C3\% A9sum\%C3\%A9s

\section{NOTES}

1. - Voir Étiquette des palais impériaux. Paris : Imprimerie impériale, 1808, chap. III, § 31-33. http:// gallica.bnf.fr/ark:/12148/bpt6k6382085v/f29.image.

2. - Sur l'histoire du Salon au XIX ${ }^{\mathrm{e}}$ siècle, voir COCHET, Vincent. «Le salon de Joséphine. La sauvegarde d'un décor déraciné ». Revue des musées de France-revue du Louvre, n 5, 2014, p. 66-77.

3. - Archives du château de Fontainebleau, Bâtiments (A.F.B.), 1808/3, année 1808-1809, état de situation.

4. - A.F.B., 1808/2, attachements de menuiserie par Leclerc, appartements de l'Empereur et de l'Impératrice, année 1808.

5. - A.F.B., 1808/3, lettre de l'intendant général de la Maison de l'empereur à l'architecte Leroy, 23 novembre 1808.

6. - Arch. nat., $\mathrm{O}^{2} 507$, dossier 5 , pièce 2 .

7. - Arch. nat., $\mathrm{O}^{2} 506$, dossier 8 , pièce 12 .

8. - A.F.B. $1808 / 3,1808-1809$, état de situation.

9. - Arch. nat., $O^{2} 506$, dossier 8 , pièce 12 et $O^{2} 508$, dossier 2 , pièce 111 .

10. - Voir BEYELER, Christophe, COCHET, Vincent. Enfance impériale : le roi de Rome, fils de Napoléon. Cat. expo., château de Fontainebleau, 26 février-23 mai 2011. Dijon : éditions Faton, 2011, $\mathrm{n}^{\circ} 43$, repr.

11. - Archives du château de Fontainebleau, Régie (A.F.R.), $\mathrm{n}^{\circ} 77$, registre des entrées 1810-1813, $\mathrm{f}$ ${ }^{\circ} 64$ et Arch. nat., $\mathrm{O}^{2} 614, \mathrm{f}^{\circ} 74$.

12. - A.F.R., $\mathrm{n}^{\circ} 217$, lettre du baron de Ville-d'Avray à Lamy, 29 mars 1816.

13. - A.F.B., 1859/1, Mémoire des ouvrages de menuiserie par Barre, 12 février-5 décembre 1859. 
14. - A.F.B., 1859/1, Mémoire des ouvrages de sculpture exécutés en carton-pierre, bois et plâtre (...) par Huber frères, 5 juillet 1859 et mémoire des ouvrages de dorure à l'huile par Desguiraud, 18 avril-15 juillet 1859.

15. - A.F.R., Inventaire de 1855, F 20363, entrée du 3 septembre 1859.

16. - A.F.R., Inventaire de 1855 , F 817.

17. - A.F.R., $\mathrm{n}^{\circ} 20227$, feuille d'entrée $\mathrm{n}^{\circ} 3$ du 27 mars 1866.

18. - A.F.R., $n^{\circ} 38024$, lettre du 30 novembre 1902.

19. - A.F.R., $\mathrm{n}^{\circ} 38044$, lettre du 18 décembre 1902.

20. - Voir DUMONTHIER, Ernest. Étoffes d'ameublement de l'époque napoléonienne. Paris : Schmid (le mobilier national), 1909, pl. 5.

21. - Inv. F 2598.2.2.

22. - Colorants Irgalane ${ }^{\circledast}$ de la firme Ciba/Huntsmann.

23. - Colorants de la gamme Unisperse de Ciba/Huntsman. Ils ont été testés et sélectionnés pour leur stabilité dans le temps et leur résistance à la lumière et à l'humidité. L'auteur de cette recherche ayant permis l'application de la technique d'impression dans le domaine de la restauration est Anne-Rose Bringel à l'occasion de son mémoire de fin d'études à l'INP-IFROA en 1997. Voir BRINGEL, Anne-Rose. «Une technique de restauration ». Dans JACQUÉ, Jacqueline et al. Histoire singulière de l'impression textile. Cat. expo, Mulhouse, musée de l'Impression sur étoffes, octobre 2000-février 2002. Aix-en-Provence : Édisud, 2001, p. 156-162.

24. - Le traitement a été mené à l'atelier ABACA Conservation-Restauration, Paris $12^{\mathrm{e}}$, en collaboration avec Émilie Énard, Judith Gauvin, Claire Roullin (restauratrices de textiles) et Déborah Panaget (restauratrice-stagiaire INP).

25. - Une collaboration a été mise en place avec l'atelier de sérigraphie professionnel Æiil de Lynx à Bagnolet, dirigé par Christophe Michel, pour la réalisation de toutes les impressions.

26. - La dépose et la pose des panneaux sont assurées par Xavier Bonnet, tapissier, Atelier Saint Louis, Paris.

\section{AUTEURS}

\section{VINCENT COCHET}

conservateur du patrimoine au château de Fontainebleau

vincent.cochet@chateaudefontainebleau.fr

\section{AGATHE STROUK}

restauratrice de textiles diplômée de l'INP (anciennement IFROA) agathe.strouk@orange.fr 